\title{
Die Seiden-Handelskompanie und Seidenraupenzucht Herzog Friedrichs I. von Württemberg
}

\author{
Von Jens Markus Schaum
}

\section{Einleitung*}

Neben seinen persönlichen und staatsmännischen Verdiensten rührt der Nachruhm Herzog Friedrichs I. von Württemberg (1557-1608) nicht zuletzt von seiner dynamischen Wirtschaftspolitik her, die sich in das durch staatliche Eingriffe geprägte Wirtschaftsmodell des Merkantilismus einordnen lässt ${ }^{1}$. Insbesondere das Montanwesen und die Textilindustrie hatten es dem eklektischen Herzog angetan und sollten gezielt weiterentwickelt werden. Sein wahrscheinlich ausgefallenstes Unterfangen stellte der Versuch dar, ein eigenes Seidengewerbe, von der Rohstoffgewinnung bis zum fertigen Produkt, in Württemberg zu etablieren.

Seidenstoffe kamen am Übergang vom 16. zum 17. Jahrhundert längst nicht mehr über die Seidenstraße aus dem Ursprungsland der Seide - China - nach Mitteleuropa. Oberitalien und in zunehmendem Maße auch Südfrankreich bildeten sich in der frühen Neuzeit als europäische Zentren der Seidenproduktion heraus. Manufakturen, technischer Fortschritt und die wesentlich kürzeren Handelswege im Vergleich zu früheren Epochen ließen die Preise für Seidenprodukte entsprechend sinken. Zwar immer noch ein relatives Luxusgut, trugen wohlhabende

* Bei diesem Beitrag handelt es sich um eine Kurzfassung meiner 2016 beim Institut für Geschichtliche Landeskunde und Historische Hilfswissenschaften der Eberhard Karls Universität Tübingen eingereichten Masterarbeit im Studienfach Geschichtswissenschaft.

${ }^{1}$ Vgl. Wilhelm SöLL, Die staatliche Wirtschaftspolitik in Württemberg im 17. und 18. Jahrhundert. Ein Beitrag zur württembergischen Wirtschaftsgeschichte, Tübingen 1934, S.13-33; Willi Alfred Boeclke, Wirtschaftsgeschichte Baden-Württembergs. Von den Römern bis heute, Stuttgart 1987, S.116ff.; Ders., Das Haus Württemberg und die Wirtschaftsentwicklung des Landes, in: 900 Jahre Haus Württemberg, hg. von Robert Uhland, Stuttgart 1984, S.636-662; Dieter Stievermann, Art. Friedrich I., in: Das Haus Württemberg. Ein biographisches Lexikon, hg. von Dieter Mertens/Volker Press/Sönke Lorenz, Stuttgart 1997, S.139-142; Paul Sauer, Herzog Friedrich I. von Württemberg 1557-1608. Ungestümer Reformer und weltgewandter Autokrat, München 2003, S. $186-300$. 
Konsumenten Seidenkleidung in nie gekanntem Ausmaß². Unsummen mussten in der Vergangenheit auch aus den Taschen der württembergischen Landeskinder für die Befriedigung des Bedarfs Seide nach Italien abgeflossen sein, aber nicht mehr länger. Die ausländischen Fertigwaren sollten in Zukunft durch eigene Erzeugnisse substituiert werden - so hatte es sich Friedrich I. von Württemberg zumindest gewünscht.

Erstmals in den Blick der Forschung geriet die Seidenunternehmung Herzog Friedrichs I. von Württemberg mit einem posthum erschienenen Aufsatz des württembergischen Finanzministers Ferdinand Heinrich August von Weckherlin in den Württembergischen Jahrbüchern. Aufgeteilt auf zwei Jahrgänge - 1831 und 1832 leistete die „Geschichte der Seiden-Cultur in Würtemberg“ absolute Grundlagenarbeit, ohne aber auf Gründe für das langfristige Scheitern der Handelskompanie und der Raupenzucht näher einzugehen ${ }^{3}$. Erste Erkenntnisse diesbezüglich brachte die Edition der „Württembergischen Landtagsakten“ (1593-1620) von Albert Eugen Adam aus den Jahren 1910-1919. Im Zuge seines Editionsvorhabens stieß Adam auf Quellen zu Herzog Friedrichs Seidenunternehmung, deren Informationen über Weckherlins Aufsatz hinausgingen. Zum einen sind diese als Landtagsakten Teil der Edition. Zum anderen präsentierte Adam diese, auf Kernaussagen reduziert, in den Fußnoten seiner Edition ${ }^{4}$. Von einer regelrechten Auswertung der

2 Ulrich Pfister, Art. Seide, in: Enzyklopädie der Neuzeit, Bd.11, Stuttgart 2010, Sp. $1043-1052$.

3 Albrecht von WeckHerLin, Geschichte der Seiden-Cultur in Würtemberg, in: Württembergische Jahrbücher für Statistik und Landeskunde (1831/32) S.116-147, hier S.116-132. Weckherlin gibt bezüglich seiner Quellen lediglich an: „Was wir berichten, ist rein aus Akten geschöpft." Bei diesen Akten handelt es sich um die beiden Büschel A 58 Bü 28 und A 248 Bü 2438 aus den Beständen des Hauptsaatsarchivs Stuttgart (HStAS). Dabei weist das Aktenbüschel A 58 Bü 28 eine Binnengliederung auf: 1. Schriften den Seidenhandel im Stock betreffend wie derselbige anzurichten war für ein Contract zwischen den Interessenten getroffen und was sonsten deßwegen für Schreiben ergangen, so aber doch zueteill kommen, und nicht bey einander. No 1-19 inct. Anno 1602 bis 1610. 2. Acta dem Seiden Handel im Stock betreffend de Anno 1606 No 1-30. Nebst einem Directorio und Subfericulo von 5 Piecen, die Rechnungen der Besoldung und Ausgaben beym Seiden Handel anbelangend. Nr. 6 fehlt. 3. Schriften die Pflanzung der Maulbeerbäume, derselbigen Erbaunng eines Hauses für die Seidenwürmer nebst 2 Rissenbelegen. Von Anno 1624-1627. Nr. 1-11 inct. Das Büschel A 248 Bü 2438 enthält drei als Hefte gebundene Handlungsbücher über die Geschäftsjahre: 23. April 1623 bis 23. April 1624, 23. April 1624 bis 23. April 1625, 23. April 1625 bis 23. April 1626. Weiterhin enthält das Aktenbüschel fünf lose, aber nummerierte, zur Seidenraupenzucht gehörende Zettel mit den Nummern 3, 4, 5, 6 und 8. Alle fünf Zettel datieren entweder in das Jahr 1624 oder 1626.

4 Albert Eugen ADAM, Württembergische Landtagsakten, Bd. 1 (1593-1598), hg. von der Württembergischen Komission für Landesgeschichte (2. Reihe), Stuttgart 1910, S.611; DERs., Württembergische Landtagsakten, Bd.2 (1599-1606), hg. von der Württembergischen Komission für Landesgeschichte (2. Reihe), Stuttgart 1911, S. 453 f.; Ders., Württembergische Landtagsakten, Bd.3 (1608-1620), hg. von der Württembergischen Komission für Landesgeschichte (2. Reihe), Stuttgart 1919, S. 434, 442-445. Adam bringt insgesamt drei 
Quellen lässt sich dabei naturgemäß nicht sprechen. Hier möchte dieser Beitrag anknüpfen, in dem der Seiden-Handelskompanie und Seidenraupenzucht Herzog Friedrichs eine Darstellung gewidmet wird, die ihren Fokus speziell auf die Fragestellung legt, warum es weder dem einen noch dem anderen Geschäftszweig gelang, sich dauerhaft zu etablieren. Dafür rentiert sich zunächst ein Blick auf die Frage, inwiefern sich die ökonomischen Vorstellungen der Zeit Herzog Friedrichs I. von Württemberg in dem Unternehmen konkret widerspiegeln.

\section{Seidenbau - eine Marktlücke des späten 16. Jahrhunderts}

Die Suche nach Inspirationsquellen für Herzog Friedrichs Seidenunternehmen führt, ähnlich wie bei seiner politischen Prägung, auf geradem Weg nach Frankreich. Beeinflusst durch die Publikation des „Théatre d'Agriculture et Ménage des Champs“ des Agronomen und „Vaters der französischen Landwirtschaft" Olivier de Serres (1539-1619) im Jahr 1600, ließ der französische König ab 1601 die wilden Bäume aus den königlichen Gärten herausreißen und Schritt für Schritt durch 14.000 Maulbeerbäume ersetzen. Die größten Maulbeerpflanzungen wurden in den Jahren 1603 und 1604 angelegt. Die Maulbeersetzlinge ließ man zusammen mit Fachleuten und einer Unmenge Seidenraupeneiern aus Italien einführen. Hinzu kamen noch speziell eingerichtete Gebäude, und die königliche Seidenmanufaktur konnte mit der Arbeit beginnen ${ }^{5}$. Die Parallele zwischen dem Seidenunternehmen Herzog Friedrichs I. und demjenigen König Heinrichs IV. liegt, wenn auch in unterschiedlichen Maßstäben, auf der Hand. Dennoch wäre es falsch, Herzog Friedrichs Seidenunternehmen als eine Nachahmung französischer Verhältnisse $\mathrm{zu}$ verstehen, was ein genauer Blick auf die Chronologie zeigt. Während der französische Hof erst 1600/1601 mit der Herausgabe des „Théatre d' Agriculture et Ménage des Champs“ eine königliche Seidenmanufaktur anstrebte und 1603/1604

neue Quellengruppen ins Spiel. Zum einen sind das die Landtagsakten selbst, die auf den sogenannten Tomi Actorum Provincialium im Ständischen Archiv Stuttgart, heute Hauptstaatsarchiv Stuttgart (Beständeserie L: Landständisches Archiv mit Landtagsarchiv), beruhen. Für diese Arbeit wesentlich ist der Band L 5 Bd. 45. Die zweite wichtige Quellengruppe Adams sind die Württembergischen Generalreskripte, in 47 Bänden gesammelt in der Ständischen Bibliothek Stuttgart, heute Handschriftenabteilung der Württembergischen Landesbibliothek (WLB) „Signatur Cod.Jur. $2^{\circ} 308^{\prime}$. Die relevanten Bände sind „Cod. jur. $2^{\circ} 308,3^{\prime \prime} 1581-1603$ und „Cod.jur. $2^{\circ}$ 308,4“ 1604-1619. Adams letzte Quelle sind die Württembergischen Landschreibereirechnungen (Bestand A 256 im Hauptstaatsarchiv Stuttgart). Im Rahmen dieser Arbeit wurden die Bände 88 (1600/01) bis 101 (1614/15) selbst gesichtet.

5 Alfred Dunder, Ausführliche Geschichte der Seidenkultur, Wien 1854, S.35-44; Hermann Grothe, Bilder und Studien zur Geschichte vom Spinnen, Weben, Nähen, Berlin 1875, S.64; Henri Silbermann, Die Seide, ihre Geschichte, Gewinnung und Verarbeitung, Bd.1, Dresden 1897, S. 90. 
mit dem Anpflanzen von Plantagen begann, ist die erste württembergische Maulbeerplantage bereits für $1595^{6}$ belegt und $1599^{7}$ sollen erste Versuche zur Seidenraupenzucht im Böblinger Schloss durchgeführt worden sein, wozu Maulbeerlaub aus Herrenberg verwendet wurde. Die Idee manifestierte sich also zuerst in Württemberg, bevor sie König Heinrich IV. in Frankreich umsetzte. Entsprechend konnte Herzog Friedrich bereits 1603, sobald sein Unternehmen Fahrt aufgenommen hatte, seinem Geheimrat Benjamin Bouwinghausen von Wallmerode drei Pfund selbsterzeugter Seidenware als Geschenk für den französischen Hof mitgeben und so auf das erfolgreiche Einführen der Seidenraupenzucht in Württemberg aufmerksam machen ${ }^{8}$.

Eine gewisse Befruchtung Württembergs durch die Entwicklung in Frankreich lässt sich dennoch konstatieren, denn die innovative Abhandlung über Seidenraupenzucht aus Serres weitgespanntem, pionierhaften Lehrbuch erregte auch das besondere Interesse Herzog Friedrichs, sodass er den fürstlichen württembergischen Kammersekretär Jakob Rathgeb (1562-1622) beauftragte, eine Übersetzung des Traktats ins Deutsche anzufertigen, die 1603 unter dem Kurztitel „Seydenwurm“ erschien. Rathgebs Vorwort zu diesem Seydenwurm-Traktat erlaubt einen Blick in die Zielvorstellungen Herzog Friedrichs: Wann auch unsere löbliche Vordern in diesem Hertzogthumb / sonderlich umb diese Statt / stetigs ibrer Altvordern Fußstapffen nachgefolt / die Wildnussen nicht außgereuttet / unnd nutzliche herrliche Weinberg und andere Einträgliche Güter zupflanzen angefangen betten / weren wir ibre Nachkommen / deß an jetzo davon habenden Nutzens

${ }^{6}$ Diese Angabe lässt sich zurückverfolgen bis auf Rudolph Moser, Beschreibung des Stadtdirections-Bezirkes Stuttgart (Beschreibungen aller württembergischen Oberämter und ihrer Gemeinden, Bd. 36), Stuttgart 1856, S.124. Eine Quelle ist dabei jedoch nicht angegeben. Der Angabe wurde in der bisherigen Forschung dennoch Vertrauen geschenkt. Vgl. Paul SAuer, Geschichte der Stadt Stuttgart, Bd. 2. Von der Einführung der Reformation bis zum Ende des 17. Jahrhunderts, Stuttgart 1993, S.131; SAuer, Herzog (wie Anm.1) S. 214.

7 Diese Angabe findet sich nur bei Rudolf Molitor, Seidenbau in Württemberg. Rückblick und Ausschau, in: Monatsblätter für den deutschen Seidenbau. April-Dezember (1932) S. 27-44, hier S.29. Eine Quelle ist dabei ebenfalls nicht angegeben. Weckherlin erwähnt diesen Sachverhalt 1831 noch nicht. Die Angabe erscheint im Großen und Ganzen dennoch glaubhaft. So schrieb Karl Pfaff: „Die erste Veranlassung dazu gaben die Privatversuche eines fürstlichen Gärtners, Seide zu erzeugen, welche nach Venedig geschickt und hier sehr brauchbar gefunden wurde." (Ders., Geschichte des Fürstenhauses und Landes Wirtemberg. Nach den besten Quellen und Hülfsmitteln neu bearbeitet, Bd.3.1, Stuttgart 1839, S.295). Beziehen sich beide Angaben auf dieselbe Quelle? Weiterhin ist die Konstellation Raupenaufzucht im Schloss Böblingen und Maulbeerlaub aus Herrenberg durchaus plausibel, weil ein Brief aus dem Jahr 1624 die gleiche Vorgehensweise erwähnt. Vgl. HStAS A 58 Bü 28, Nr.3.

${ }^{8}$ HStAS A 58 Bü 28, Nr. 9, 12. Vgl. Weckherlin (wie Anm. 3) S. 121; Adam (wie Anm. 4, Bd.2) S.453f., Anm.2; Gerhard Hertel, Herzog Friedrich I. von Württemberg. Eine geschichtliche Erzählung, Horb am Neckar 1989, S. 120; SAuER, Herzog (wie Anm. 1) S. 214. 
unnd wolgefälligen Eintrags / auch beraubt. Dann es muß alle ding einen Anfang haben. Dieweil dann Gnädiger Fürst unnd herr / auß obgebörten / zwar in Eyl / und schlecht / von mir (als einem der dises Handels / ausserbalb was er gelesen / noch nicht zum besten erfabren) eingeführten Ursachen / ein solch hoch nutzliches Seydinwerck in Ewer Fürstlichen Gn. Löblichen Hertzogthumb gar wol / obne einigen Schaden / oder Verhinderung anderer Feldtgeschefften / mit Lob unnd Nutzen angerichtet werden mag / Ewer Fürstl. Gn. Auch als ein Christenlicher Fürst und sorgfeltiger Vatter deß Vatterlandts / allbereit mit nicht geringem Costen / den Anfang unnd die gewisse unverwerffliche Prob gemacht / beneben selbiges sondern allen zweiffel dero Unterhanen unnd Angebörigen zum guten Exempel / Nutzen unnd Eintregligkeit / also zu continuieren / unnd unablässig fortzusetzen geneigt seind ${ }^{9}$.

Herzog Friedrichs Seidenunternehmen begriff sich folglich als Pionierbetrieb, als Vorbild für alle Landeskinder, um den Seidenbau in Württemberg genauso heimisch zu machen wie den Weinbau ${ }^{10}$. Auch der französische König Heinrich IV. bemühte sich nach Kräften, die Seidenraupenzucht unter der Landbevölkerung zu fördern. Die Landleute erhielten unentgeltlich Maulbeersetzlinge, Raupeneier und Unterricht in der Seidenzucht, sodass Frankreich 1606 schon um die vier Millionen Maulbeerbäume verfügte ${ }^{11}$. In Württemberg belief sich die Anzahl an Maulbeerbäumen nach dem Tod Herzog Friedrichs I. 1608 immerhin auf mehrere Tausend ${ }^{12}$.

Der Kerngedanke beider Herrscher lässt sich als jene Neubetrachtung des Außenhandels auffassen, die Eli Filipp Heckscher als Übergang von einer Versorgungspolitik hin zu einer Schutzpolitik formulierte. Das staatliche Augenmerk war nicht mehr darauf gerichtet, der Bevölkerung eine ausreichende Seideneinfuhr für ihre Konsumtion zu gewährleisten, sondern die Produktion des eigenen Seidenbedarfs selbst in die Hand zu nehmen - praktisch eine Marktlücke zu schließen - und nach Möglichkeit sogar selbst zum Seidenexporteur aufzusteigen ${ }^{13}$. Anders formuliert fand hier in Kombination mit dem merkantilistischen Fixpunkt, dem Festhalten an Geld und Edelmetallen und der Angst, sie zu verlieren, die Neubetrachtung des Außenhandels als Nullsummenspiel statt, bei dem der Importeur verliert, was der Exporteur gewinnt. Der Exportüberschuss galt als Idealfall,

9 Johann Jacob Rathgeb, Seydenwurm. Von Art, Natur, Eigenschafft und grosser Nutzbarkeit dess edlen Seydenwurms, Tübingen 1603, fol. 5 r. Herzog Friedrich bestellte für sein Seideninstitut sogleich mehrere Exemplare, die er sich immerhin $21 \mathrm{fl}$. kosten ließ. Vgl. Adam (wie Anm. 4, Bd.2) S. 611.

10 Vgl. WLB Cod.Jur. $2^{\circ} 308,3$ - 13. November 1601; HStAS A 58 Bü 28, Nr.1.

11 Dunder (wie Anm. 5) S. 40; Grothe (wie Anm. 5) S. 63 f.

12 HStAS L 5 Bd. 45, fol. 424 r; AdAm (wie Anm. 4, Bd. 3) S. 444.

13 Eli Filip Heckscher, Der Merkantilismus, Jena 1932, Bd.2, S.67-130. Vgl. Rathgeb (wie Anm.9) fol.3r; Rainer Gömmel, Die Entwicklung der Wirtschaft im Zeitalter des Merkantilisums 1620-1800 (Enzyklopädie Deutscher Geschichte, Bd.46), München 1998, S.41-56; Thomas Sokoll, Art. Merkantilismus, in: Enzyklopädie der Neuzeit, Bd.8, Stuttgart 2008, Sp. 380-387. 
um die finanziellen Mittel zur Entwicklung des eigenen Staates auf Kosten eines anderen Staates zu erhalten. Statt der eigenen Konsumtion, stellte die Schutzpolitik somit die eigene, möglichst große Produktion in den Fokus, um das Ziel einer aktiven Handelsbilanz zu erreichen.

Die ersten Maulbeerbäume musste der Herzog zwangsläufig aus Italien einführen ${ }^{14}$. Damit sich eine so teure Investition in Zukunft nicht mehr wiederholte, legte Friedrich I. am Produktionsort Stuttgart in unmittelbarer Nähe zur Residenz zwei maulböhr bahm mutter lender [Baumschulen] das eine hündern Jäger Hauß unnd das ander bey dem Acht Eckbeten Thurn an, damit jedes Jahr etliche hundert Maulbeerbäume daraus versetzt werden konnten ${ }^{15}$. Bei den Seidenraupeneiern hingegen verließ man sich nicht nur auf die eigene, „Landsamen“ genannte Nachzucht, sondern kaufte auch noch in späteren Jahren italienischen und spanischen Samen hinzu ${ }^{16}$. Die Maulbeerplantagen legte man offenbar in Etappen an. Eine verständliche Vorgehensweise, wenn in der Baumschule erst neue Schößlinge herangezogen werden sollten. Insgesamt sind drei Plantagen bekannt: $1595 \mathrm{im}$ Lustgarten $^{17}$, 1600 auf dem Gelände des trockengelegten Oberen Sees ${ }^{18}$ und 1608 auf der Baumreute im Eiernest ${ }^{19}$, was die Existenz weiterer, nicht überlieferter Plantagen in Stuttgart keineswegs ausschließ $\mathrm{t}^{20}$. Um das Unternehmen auch mit Räumlichkeiten für die Aufzucht der Seidenraupen und das Spinnen, Färben und Weben der Seidenfäden zu versehen, stellte Herzog Friedrich das sogenannte „Stockgebäude“ in Stuttgart zur Verfügung. Nach Abriss und Neubau im 19. Jahr-

${ }^{14}$ HStAS L 5 Bd.45, fol.424 r; Adam (wie Anm.4, Bd.3) S.444. Vgl. Hertel (wie Anm. 8) S.120; Sauer, Herzog (wie Anm. 1) S. 214.

15 HStAS A 58 Bü 28, Nr.1. Vgl. Weckherlin (wie Anm.3) S. 124.

16 HStAS A 58 Bü 28, Nr. 3, 9. Vgl. Weckherlin (wie Anm. 3) S. 121 f.; Söll (wie Anm. 1)

S. 27; SAUER, Herzog (wie Anm. 1) S. 214.

17 Moser (wie Anm. 6) S. 124.

18 Diese Angabe ließ sich zurückverfolgen bis auf Gustav WaIs, Alt Stuttgart. Die ältesten Bauten, Ansichten und Stadtpläne bis 1800 (Veröffentlichungen des Archivs der Stadt Stuttgart, Bd. 8), Stuttgart 1941, S. 70: „Von der 1600 auf dem Gelände des Oberen und 1752 auf dem Mittleren Sees vorgenommenen Anpflanzung von Maulbeerbäumen erhielt die Gegend die Bezeichnung ,In den Seidengärten', ebenso die Seidenstraße ihren Namen." Eine Quelle ist dabei jedoch nicht angegeben. Der Angabe wurde in der bisherigen Forschung dennoch Vertrauen geschenkt. Vgl. SAUER, Geschichte (wie Anm.6) S.131; Sauer, Herzog (wie Anm.1) S.214.

19 HStAS A 403 II Bü 21 Nr.1-4. Das Gelände für die jüngste Plantage „auf der Baumreute im Eiernest" musste Herzog Friedrich erst von dem Mundschenk Christoff Mockheln für 237 fl. abkaufen. Vgl. SAUER, Geschichte (wie Anm. 6) S. 131; SAUER, Herzog (wie Anm.1) S. 214.

20 WeCKHerlin (wie Anm.3) S.124 ging noch von einer Plantage „wahrscheinlich im Schloßgarten“ aus. Karl Pfaff, Geschichte des Fürstenhauses und Landes Wirtemberg. Nach den besten Quellen und Hülfsmitteln neu bearbeitet, Bd.3.1, Stuttgart 1839, S. 295 f. wusste schon von zwei Maulbeergärten in Stuttgart zu berichten. Erst Paul Sauer sichtete das Büschel A 403 II Bü 21 im Hauptstaatsarchiv Stuttgart und entdeckte damit die dritte Plantage „auf der Baumreute im Eiernest“; Ders., Geschichte (wie Anm.6) S. 131. 
hundert steht an derselben Stelle heute die Neue Kanzlei ${ }^{21}$. In der Umgangssprache der Zeitgenossen am württembergischen Hof wurde der Stock geradezu synonym mit dem Seidenunternehmen als Ganzes ${ }^{22}$.

Während der Initialphase des Seidenunternehmens und darüber hinaus betraute Herzog Friedrich drei Männer mit der Verwaltung beziehungsweise Geschäftsführung im „Stock“: Wolff Ganß, Cesar Morello, und Buchballter do. Eberhardt $B e b r^{23}$. Wolfgang Gans (1563-1636) war als Organist an der Stuttgarter Stiftskirche und bei Hof tätig, sowie seit 1624 Gewölbeverwalter. In den Quellen zum Seidenunternehmen taucht er vor allem im Zusammenhang mit den Frankfurter Messen auf ${ }^{24}$. Cäsar Morell († 1626?) brachte Herzog Friedrich von seiner Reise nach England 1592 mit. Er diente Herzog Friedrich I. im Anschluss als Leibdiener. Beim Stock fiel ihm der Einkauf von Seidenraupeneiern, Rohseide und in späteren Jahren fertiger Seidenware aus Italien als besonderer Zuständigkeitsbereich $\mathrm{zu}^{25}$. Dr. jur. Eberhard Beer ( $†$ 1624) war bis zum Jahr 1622 Buchhalter des Seidenunternehmens und von 1622 bis zu seinem Tod 1624 fürstlicher Gewölbeverwalter ${ }^{26}$. Die Rolle eines „Oberinspektors“ übte Benjamin Bouwinghausen von Wallmerode aus ${ }^{27}$. Der breite Mitarbeiterstab beim „Stock“ wird in den Quellen als „Laboranten" bezeichnet ${ }^{28}$.

In die Zusammensetzung des Mitarbeiterstabs erlauben die Quellen folgenden Einblick $^{29}:$ 1. Der Maulbeerbaum-Gärtner Johann Kornmesser ${ }^{30}$, 2. Der Meister

${ }^{21}$ HStAS A 58 Bü 28, 1 Nr. 2 (Contract des seidinhandels halb, $\ 4$ ). Vgl. WeCKHERLIN (wie Anm.3) S.121 f.; Adam (wie Anm.4, Bd.2) S.453, Anm.2; Wais, Alt Stuttgart (wie Anm.18) S. 102 f.; Gustav WAIS, Alt Stuttgarts Bauten im Bild, Stuttgart 1951, S.24, 203; Sauer, Herzog (wie Anm. 1) S. 214.

22 Einige Beispiele: HStAS A 256 Nr. 95, fol. 149 r; HStAS A 256 Nr. 97, fol. 146 r; HStAS: L 5 Bd 45 fol. 419 r-fol. 425 r; AdAm (wie Anm. 4, Bd.3) S. 442-445; HStAS A 58 Bü 28, Nr.26, 30 .

${ }^{23}$ HStAS A 58 Bü 28, Nr. 2 (Contract des seidinhandels halb, $\mathbb{} 3$ ).

${ }^{24}$ HStAS A 58 Bü 28, Nr.10-14. Vgl. Walter Bernhardt, Die Zentralbehörden des Herzogtums Württemberg und ihre Beamten 1520-1629, Bd.1 (VKgL B 70), Stuttgart 1972, S.310f.

${ }^{25}$ HStAS A 58 Bü 28, Nr. 23 und Nr. 9. Ein auf den 5. Januar 1627 zu datierender Brief erwähnt, dass Cäsar Morell gestorben war. Vgl. AdAm (wie Anm.4, Bd.3) S. 442, Anm. 5; BERnHARDT (wie Anm. 24) S. 511.

26 Adam (wie Anm. 4, Bd.3) S. 442, Anm. 4; Bernhardt (wie Anm. 24) S. 146.

27 Bernhardt (wie Anm. 24) S. 179 ff.; Weckherlin (wie Anm.3) S. 122.

${ }_{28}$ Das hervorstechendste Merkmal der Laboranten scheint, zumindest nach Meinung Herzog Friedrichs, ihr obnfleis und saumnus, beneben andern mängeln gewesen zu sein und jeder nur seines gefallens handlen möchte, weswegen Beniamin Buwingkhausen von Wallmerode, auch Wolff Gans und Cäsar Morello als Aufseher über die Laboranten fungierten und ihnen weisungsbefugt waren. Vgl. HStAS A 58 Bü 28, Nr. 5; HStAS A 58 Bü 28, Nr. 2 (Contract des seidinhandels halb, $\mathbb{S} 9$ ).

${ }^{29}$ Vgl. auch die Auflistung bei Weckherlin (wie Anm. 3) S. 122.

30 HStAS A 58 Bü 28 Stapel 2 Nr. 16; HStAS A 58 Bü 28 Stapel 3 Nr. 3, 6, 10. 
für die Seidenwürmer Jacomo di Marchi ${ }^{31}$, 3. Ein Meister und eine Meisterin niederländischer Herkunft für das Seidenspinnen ${ }^{32}$, 4. Der Seidenfärber Philibert Corwin ${ }^{33}$, und 5. Der „Seidenbereiter“ Johann Schott ${ }^{34}$, der ab 1622 die Funktion des Buchhalters von Dr. Eberhard Beer übernahm und zudem in den 1620er Jahren die Geschäftsführung der Seidenraupenzucht ausführte ${ }^{35}$. Weiterhin sind einige italienischstämmige Facharbeiter namentlich überliefert ${ }^{36}$. Neben den Geschäftsführern und den Laboranten, setzte sich der Mitarbeiterstab noch aus einer dritten, sehr speziellen Gruppe für die Hand-und-Spanndienste zusammen, den „Armen Kindern" 37 .

Am 13. November 1601 verfügte Herzog Friedrich in einem Generalreskript, dass zur Emporbringung des württembergischen Seidenbaus von jeder Stadt und jedem Amt jährlich zwei Jungen oder Mädchen, welche ohnehin im Müßiggang von den Armenkästen und Spitälern erhalten werden müssten, zur Erlernung des Seidenspinnens nach Stuttgart geschickt und für diese Armen Kinder so lange bis sie ihren Unterhalt selbst verdienen können, je $20 \mathrm{fl}$. Kostgeld bezahlt werden solle $^{38}$. Zusätzliche $15 \mathrm{fl}$. Kostgeld pro Kind und die konkrete Versorgung der insgesamt 53 Kinder mit allem Lebensnotwendigen versprach der Herzog dafür auf sich zu nehmen ${ }^{39}$. Eine Maßnahme, die Friedrich I. als Gewinn für alle Beteiligten darstellte: dieweil dises mehister theyls den armen zu guetem, und irer beßerer $u ß-$ bringung angesehen, welche sonsten wie es die tägliche erfahrung giebet im müßiggang uffwachsen, den armen spittälen, cästen auch sonsten männiglich beschwerlich, solchem aber bierdurch guten theils abgeholffen werden kann ${ }^{40}$.

${ }^{31}$ HStAS A 58 Bü 28 Stapel 2 Nr.15; HStAS A 256 Bd. 93 fol. 319 v.

32 WLB Cod.jur. fol. 306-3; HStAS A 58 Bü 28, Nr. 24, 28, 29.

33 HStAS A 58 Bü 28, Nr.24.

${ }^{34}$ HStAS A 58 Bü 28, Nr. 24.

35 HStAS A 58 Bü 28, Nr. 4.

36 Johann Maria Jardiniero, Andrea Rocca Dimorari, Johann Bernhardo Bezi, Martin Collogaro, Fabian Compani, Camilla, Bernhardo Bettis Wittib und Margareta Fassata. HStAS A 58 Bü 28, Nr. 4 und Nr. 8, 19; HStAS L 5 Bd. 45 fol. 419 v; Vgl. Adam (wie Anm. 4, Bd.3) S.537, Anm.3; Adam (wie Anm.4, Bd.3) S.443; Weckherlin (wie Anm.3) S.122; Hertel (wie Anm. 8) S.120; Sauer, Herzog (wie Anm. 1) S. 214.

37 Die Namen der Kinder sind in einem Summarische[n] verzeichnuß überliefert (HStAS A 58 Bü 28, Nr.24). Diese Namen hier aufzulisten würde aber den Rahmen sprengen.

38 WLB Cod.jur. $2^{\circ}$ 308,3-13. November 1601. Die Konzeptversion ist Teil der Aktenüberlieferung. Vgl. HStAS A 58 Bü 28, Nr. 1. In Kurzform findet sich das Generalreskript auch bei August Ludwig ReYscher (Hg.), Vollständige, historisch und kritisch bearbeitete Sammlung der württembergischen Gesetze, Bd.12, Tübingen 1841, S. 585.

${ }^{39}$ HStAS A 58 Bü 28, Nr.14, 24. Im Generalreskript wird dieser Sachverhalt nicht erwähnt.

40 WLB Cod.jur.2 ${ }^{\circ}$ 308,3-13. November 1601. Vgl. Adam (wie Anm. 4, Bd.2) S. 453 f., Anm.2; Weckherlin (wie Anm.3) S.122f.; Söll (wie Anm.1) S.27; Hertel (wie Anm.8) S. 120; Sauer, Herzog (wie Anm. 1) S. 214. 
So malerisch wie sich Herzog Friedrich dieses Szenario vorstellte, verlief es in der praktischen Umsetzung nicht. Zu dieser Maßnahme trieben ihn auch nicht der pure Altruismus, sondern vielmehr handfeste wirtschaftliche Überlegungen. In engem Zusammenhang mit den handelspolitischen Zielsetzungen stand der Gedanke an eine Beschaffung von Arbeitsgelegenheiten im Inland. Dementsprechend schreibt Jakob Rathgeb im Vorwort seines Seydenwurm-Traktats: Wann die Bäum allein anfangs gepflanzt und erwachsen sein / daß man keinen Mangel an Blättern hat / kann diese Arbeit / in Siben oder Acht Wochen / von schlechten unarbeitsamen unnd solchen Leuthen / die sonsten ein Pflug zu heben / oder einen Karßt zu fübren / vil zu schwach / wol vollendet / ja gar bis auff das ausspulen / gefertiget und verrichtet werden ${ }^{4}$.

Mit einem württembergischen Seidengewerbe versprach man sich also Leute in Lohn und Brot zu bringen, die anderweitig keiner Erwerbstätigkeit nachgehen konnten. Die Erhöhung der Beschäftigungszahlen sollte die „consumtio interna“ ausweiten und außerdem Druck auf die Löhne ausüben, womit die Erzeugnisse im Preis sanken und auf den internationalen Märkten wettbewerbsfähig blieben ${ }^{42}$.

Wie beim Parallelinstitut am französischen Königshof war auch Herzog Friedrichs Seidenunternehmen zunächst auf Selbstbetrieb hin konzipiert, wollte also die eigene Rohseide weiterverarbeiten ${ }^{43}$. Die Menge an selbst erzeugter Rohseide, die in den Quellen mit „Landseide“ tituliert wird, erlaubte in den Anfangsjahren allerdings keinen nennenswerten Handel ${ }^{44}$. Quittungen sind für die Jahre 1602 (7 lib. 11 lot) und 1606/1607 (11 lib. 11¹/2 lot) überliefert ${ }^{45}$. Im Vergleich dazu nahm die fürstliche Gewölbeverwaltung in späteren Jahren zwischen 30 und 40 Pfund Seide allein zur jährlichen Eigenbedarfsdeckung $\mathrm{an}^{46}$. In jedem Fall hinkte die Rohseidenproduktion den Verarbeitungskapazitäten des Stocks hinterher. Für dieses Dilemma boten Wolfgang Gans und Cäsar Morell am 16. Januar 1602 einer im Stockgebäude versammelten Kommission bestehend aus Erhardt Stickel, Johann Wilhelm Breitschwerdt und Jakob Rathgeb eine Lösung an, die in folgender Relation protokolliert wurde: Lieber gnediger fürst und herr, uff Euer Fürstlichen Gnaden gnedigen

41 Rathgeb (wie Anm. 9) fol. 4 v.

42 Blaich, Die Epoche des Merkantilismus, Wiesbaden 1973, S. 90-94; Gömmel (wie Anm.13) S. 47 f.; Rolf Walter, Wirtschaftsgeschichte. Vom Merkantilismus bis zur Gegenwart. (Wirtschafts- und Sozialhistorische Studien, 4), Köln/Weimar/Wien ${ }^{32000, ~ S .29 f . ~}$

43 Weckherlin (wie Anm.3) S. 121.

${ }^{44}$ Ein Decretum Seydinhandels Im Stockh betreffendt. Wie die verbesserungs mittel ins werck zu richten von 1610 bemerkt hierzu: Was nun erstlich Irer $F$. G. aigne landtseidin betrifft, Befünden Ire F.en G.en das derselbigen wenig gnug, dannenhero Iren F.en G.en ein usfürlich bedencken, wie solche zuvermehren, und zu bestem eintrag zurichten, durch gedachten Rentcammerrhat Moser fürderlich gegeben werden soll [...] (HStAS A 58 Bü 28, Nr. 19).

${ }^{45}$ HStAS A 58 Bü 28, Nr. 4, 9.

${ }^{46}$ HStAS A 248 Bü 2438: Rechnung von Georgii Anno 1623 biß Georgii Anno 1624, Rechnung von Georgii Anno 1625 biß Georgii Anno 1626; HStAS A 58 Bü 28, Nr. 10. 
bevelch, haben wir uns an heut in Stockh verfuegt, uns in denen durch Wolff Gansen und Cäsar Mourel vorgezeigten rechnungen und uberschlägen mit fleiß erseben. Und sovil befunden, das es unserm geringen verstandt nach ein herrlicher löblicher unnd einträglicher handel. Dan erstlich würdt vermög baigelegten extracts das pfund rober seiden zu 32 lot zu Venedig erkhaufft mit zoll fubrlobn und allen uncosten bißhirher gebn Stuttgartt gebracht umb-4 fl. $9 \mathrm{kr} .4 \mathrm{hlr}$. Im filatorio in der farb und biß allerdings zum incartieren costs ungevehr - $53 \mathrm{kr}$. 1. hlr. thuet das pfund in allem $5 \mathrm{fl}$. $13 \mathrm{kr}$. macht ein centner - $521 \mathrm{fl}$. $40 \mathrm{kr}$. Solche seiden würdt albie uffs geringst das pfund umb 7 gulden gegeben. Thet der centner -700 gulden. Webr also uff jedem centner nach zugewin und uberschatz ungevehr $178 \mathrm{fl}$. $2 \mathrm{kr}$. Dieser roher seiden möchten wan das werckh ein wenig in gang gebracht, jebrlich uff 30 centner verarbeit und vertriben werden weil es sebr schöne und gute kauffmans wabr, daruff hatte man uber allen uncosten zugewin 5.399 fl. 20 batzen ${ }^{47}$.

Wolfgang Gans und Cäsar Morell bewiesen mit diesem Konzept ihr ökonomisches Fingerspitzengefühl. Denn dem Außenhandelsprimat von der Förderung des Exports bei gleichzeitiger Eindämmung von Einfuhren fügte einige Jahre zuvor Jean Bodin (1529-1596) mit einer seiner ökonomischen Kernthesen eine Ergänzung hinzu. Er postulierte in seinen „Six Livres de la République“, dass ein Import von Rohstoffen und ein Export von Fertigwaren zu empfehlen bzw. ein Export von Rohstoffen und ein Import von Fertigwaren zu vermeiden sei: Et quant aux matieres qu'on apporte des pays estrangers, il est besoin de rabbaisser l'impost, E le hausser aux ouvrages de main, Ene permettre qu'il en soit apporté de pays estrange, ni soffrit qu'on emporte du pays les denrees cruës, comme fer, cuyure, acier, laines, fil, soye crüe, E autres matieres semblables: à fin que le subiect gaigne le profit de l'ouvrage, le Prince l'imposition foraine ${ }^{48}$.

Diese These muss im engen Zusammenhang mit dem Gedanken der Arbeitsbeschaffung gesehen werden. Beim Kauf einer ausländischen Fertigware wird nicht nur der Materialwert bezahlt, sondern auch die aufgebrachte Arbeitsleistung und der Kapitaleinsatz. Diese Wertschöpfung wollten die Merkantilisten natürlich gerne in ihrem Land erbracht sehen und nicht an das Ausland verlieren. Das Ausland sollte die billigen Vorprodukte liefern, wohingegen man selbst die teuren Endprodukte verkaufen wollte ${ }^{49}$. Besonders interessant an der Textstelle aus Jean Bodins Werk ist, wie sehr sich hierin Herzog Friedrichs Wirtschaftspolitik als Ganzes widerspiegelt. Die aufgezählten Rohstoffe lassen sich in zwei Gruppen einteilen zum einen Metalle: fer (Eisen), cuyvre (Kupfer) \& acier (Stahl) und zum anderen Textilien: laines (Wolle), fil (Garn) \& soye crüe (Rohseide). Diesen beiden Bereichen, Montanwesen und Textilindustrie widmete Herzog Friedrich seine größte

47 HStAS A 58 Bü 28, Nr.1.

48 Jean Bodin, Les Six Livres de la République. Avec l'Apologie de René Herpin (Faksimiledruck der Ausgabe Paris 1583), Aalen 1961, S. 877.

49 Gömmel (wie Anm.13) S.46ff.; Walter (wie Anm.42) S.27-30; Sokoll (wie Anm. 13) Sp. $382 \mathrm{ff}$. 
Aufmerksamkeit und er bemühte sich, jeweils die größtmögliche Wertschöpfung in Württemberg zu erreichen: erst durch eine Ausweitung der Rohstoffbasis (Erhöhung der Erzfördermengen, Steigerung des Flachs und Hanfanbaus), weiter durch eine Erhöhung der inländischen Verarbeitung (Bergfreiheit, Leinenweberzunft) und schließlich durch einen Schutz der einheimischen Märkte vor ausländischer Konkurrenz (Eisenmonopol, Leinenweberordnung).

Fernhandel stellt vergleichsweise hohe Ansprüche an das eingesetzte Kapital ${ }^{50}$, weswegen Wolfgang Gans und Cäsar Morell in ihrer Relation folgendermaßen fortfuhren: Es werden aber, zur verlag unnd einkhauffung derselbigen [Rohseide] uff ungevehr 16.000 gulden erfordert sein. Wie und welcher gestalt euer gnaden ein solches werckh verlegen, vortreiben, unnd was sie hiebrunder ferners verordnen wollen, das steth zu dero gnedigen unnd fürderlichen resolution, allderweil die messen herzu ruckben und man sich (damit die arbeutter nit müssig gehen dörffen, sonder das werckh starckh vortgetriben werden khonne) mit roher seiden versehen unnd gefasst machen mu $\beta^{51}$. Seinem Naturell entsprechend, entschied sich Herzog Friedrich für die zeitgemäße Herangehensweise, das heißt zur Gründung einer Handelskompanie. Mit Beginn des 17. Jahrhunderts verbanden Kapitalgesellschaften immer häufiger staatliche Privilegierung mit einem eigenen Kapitalstock und in den meisten Fällen auch einer eigenen Handelsorganisation. Das Kapital stammte dabei nicht allein von Kaufleuten, sondern auch von Beamten und Adeligen. Auf diese Weise gelang sowohl eine Ausweitung der Kapitalbasis als auch eine Interessenverbindung mit der Herrschaft ${ }^{52}$. Damit lässt sich als Abschluss dieses Kapitels festhalten, dass Herzog Friedrichs Seidenunternehmen mit seinen beiden Geschäftszweigen Handelskompanie und Raupenzucht die konkrete Umsetzung merkantilistischer Ökonomie, im Speziellen der Ökonomie Jean Bodins, darstellt. Die zwei folgenden Kapitel untersuchen nun, wie sich diese Geschäftszweige in der Praxis schlugen.

\section{Die Seiden-Handelskompanie}

Am 23. April 1602 setzte Herzog Friedrich zusammen mit seinen sieben Mitinteressenten: den beiden Kammersekretären Johann Sattler und Jakob Rathgeb, den beiden Landschreibereiverwaltern Erhardt Stickel und Johann Wilhelm Breitschwerdt, dem Hoforganisten Wolfgang Gans, dem Kammerdiener Cäsar Morell sowie dem Hofrat Benjamin Bouwinghausen von Wallmerode die Geschäftsidee

50 Heckscher (wie Anm.13) S.309f.; Gömmel (wie Anm 13) S. 81.

51 HStAS A 58 Bü 28, Nr.1.

52 Heckscher (wie Anm.13) S.309-317; Hermann Kellenbenz, Art. Handelsgesellschaft, in: LexMA, Bd.4, München 1999, Sp. 1901; Markus A. Denzel/Ulrich Pfister, Art. Handelsgesellschaft, in: Enzyklopädie der Neuzeit, Bd.5, Stuttgart 2007, Sp. 97-101. 
Seiden-Handelskompanie mit einem Contract des seydinbandels halb in die Tat $u^{53}$. Startkapital waren allerdings nur $15.000 \mathrm{fl}$, da Cäsar Morell nur $1.000 \mathrm{fl}$. beisteuerte ${ }^{54}$. Über dieses eingesetzte Kapital sollte jedes jars uff Georgii, also dem Sankt Georgstag (23. April), ordenliche und uffrechte rechnung gethon werden. Nach Ablauf der zwölfjährigen Vertragslaufzeit galt es, das eingelegte Kapital wieder auszubezahlen. In seiner weiteren Konzeption wirkt der Contract des seydinhandels halb um einen Ausgleich zwischen den beiden Polen - Herzog auf der einen Seite und Mitinteressenten auf der anderen Seite - bemüht. Den fürstlichen Vergünstigungen werden möglichst gleichwertige Verpflichtungen gegenübergestellt. Als Vergünstigung verbot Herzog Friedrich dergleichen handtierung mit außländischer seydin [...] obne sonderbares vorwißen und einwilligen obgesetzter contrabenten und mitintereßenten während der nächsten zwölf Jahre im Herzogtum Württemberg. In gleicher Manier gewährte er jegliche Steuer- und Abgabenfreiheit, überstellte das Stockgebäude für zwölf Jahre an die Handelskompanie und genehmigte die kostenlose Brennholzversorgung des Stocks im ersten sowie die kostengünstige Versorgung von $2 \mathrm{fl} .16 \mathrm{kr}$. das Klafter Brennholz in den folgenden Jahren. Als Verpflichtung nahm der Herzog die Versorgung der Armen Kinder nur in den ersten drei Jahren auf sich, danach musste die Versorgung durch die Handelskompanie erfolgen. Auch der Meister und die Meisterin zum filatorio, die gleichzeitig für die Ausbildung der Armen Kinder zuständig waren, sollten nur für drei Jahre aus der herzoglichen Kasse unterhalten werden. Den Ertrag aus der selbst erzeugten Landseide sprach sich der Herzog selbst zu, sowie ab dem zweiten Geschäftsjahr den neunten Teil vom Gewinn aus der verarbeiteten Seide und zusätzlich für jeden verarbeiteten Ballen einen königlichen hispanischen Thaler (50 fl.) an Hauszins. Der Einkauf der Färbemittel und die Erhaltung der Werkzeuge waren aus dem allgemeinen Handelsaufkommen zu erbringen. Die scheinbare Ausgeglichenheit zwischen Vergünstigungen und Verpflichtungen erwies sich jedoch als trügerisch.

Über das erste Geschäftsjahr der Handelskompanie (23. April 1602 bis 23. April 1603) lassen sich praktisch keine Aussagen treffen. Ein Brief legt jedoch den Schluss nahe, dass sich die Verfertigung im Stock erst einmal konsolidieren musste, so hieß es, dass noch zur zeit, (wie mich anlangt) allein schnier unnd portenn gewirckht werden, daß auch die strickberey vonn allerband seydenn, atlassen unnd wullen strimpffen, hemden, hendschuben, hauben neben dem seyden handel nit übel

${ }^{53}$ HStAS A 58 Bü 28, Nr. 2. Der Vertrag zur Gründung der Seiden-Handelskompanie liegt nur als Konzept auf neun doppelseitig beschriebenen und gefalteten Briefbögen mit zahlreichen Durchstreichungen und Ergänzungen vor. Dabei fertigte die Kanzlei, wie es am Ende des Textes erwähnt wird, acht gleichlautende Originale auf Pergament, versehen mit den Unterschriften Herzog Friedrichs I. und seiner Mitinteressenten respective fürstlichem secret und innsigeln, wovon jede der Vertragsparteien eines überstellt bekam.

${ }^{54}$ HStAS A 58 Bü 28, Nr.7: Und dieweil darunder den 7. theilen von wegen Cäsars $1.000 \mathrm{fl}$. weniger alls der andern mitinteressenten. 
stüende ${ }^{55}$. Bis zum 24. Mai 1603 scheint die Verarbeitung im Stock jedoch insoweit angelaufen zu sein, dass Herzog Friedrich über ein Generalreskript daran erinnern konnte, daß albereit ein ansebnliche anzabl guther feiner und anderer seiden, wie auch bortten, schnüre, und sonsten seidener zeng, von allerley farben daraus bereith und zugericht und wir also in guther hofnung standen derselbe je länger je mebr in aufgang gebracht und zunehmen werde. Damit wir dann darinnen desto mebrern vertrieb gehaben, und unsere unterthanen (so deren bedurftig) auch fruchtbarlich genüssen mögen. So ist hiemit unser befelch, $d u$ wöllest solches alles den handels leuthen und krämern, in deiner anbefoblenen ampts verwaltung, so mit dergleichen wabren umb gehen, und handthieren, fürhalten und sie dabin erinnern, dieweil sie dieselbe bey berührtem handel, mit bessere gelegenheith, und wobl failerem wehrt, als auserbalb landts, zu wegen bringen, undt bekommen könten, daß sie fürobin solche seide wabren zu erstehung mehr mühe und ohncostens, bey besagtem handel alhie erkaüf und so wohl in befürderung ibres als unsers nuzens sich darnach richten thuen daran beschibt unser gnädiger will und meinung ${ }^{56}$. Neben einem Blick auf die Produktpalette erlaubt dieses Generalreskript Rückschlüsse auf den Vertrieb der Seidenhandelskompanie. Der Stock erscheint hier nämlich zunächst einmal als eine Art Großhändler, der seine Ware an handels leuthe und krämer weiterverkauft $^{57}$. Passenderweise bewegte man sich auf den Frankfurter Messen, um zum Beispiel Färbemittel und mindestens einmal sogar Rohseide einzukaufen sowie um fertige Seidenwaren zu verkaufen ${ }^{58}$.

In Bezug auf die Profitabilität der Handelskompanie für den Herzog einerseits und die Mitinteressenten andererseits erweist sich eine Rechnung im seydinhandel sine dato, die sich allerdings mit einiger Gewissheit in das vierte Geschäftsjahr (23. April 1605 bis 23. April 1606) verorten lässt ${ }^{59}$, als besonders aufschlussreich. Zunächst zum Inhalt dieser einzigen erhaltenen Jahresabschlussrechnung: Aus dem Stammkapital von 15.000 fl. konnte die Handelskompanie in dem fraglichen Jahr einen Ertrag von 1.093 fl. 32 kr. erwirtschaften. Dabei verteilte sich das Kapital auf folgende Posten: anschlag der waren und species $12.305 \mathrm{fl} .251 / 2 \mathrm{kr}$. - schulden laut der specification $1.793 \mathrm{fl}$. $131 / 2 \mathrm{kr}$. - baargelt $1.994 \mathrm{fl} .53 \mathrm{kr}$. Aus dem erwirtschafteten Ertrag gebührten Herzog Friedrich der Hauszins von 50 fl., das Entgelt von 90 fl. 40 kr. für 40 Klafter Brennholz à 2 fl. 16 kr. und eine Verarbeitungspauschale von 4 fl. $12 \mathrm{kr}$. für drei Ballen verarbeiteter Rohseide. In Summe macht dies 144 fl. 52 kr., die von dem erwirtschafteten Ertrag abzuziehen waren. An die Interessenten konnten somit 948 fl. 40 kr. aus dem Bargeldbestand als Gewinn verteilt

\footnotetext{
55 HStAS A 58 Bü 28, Nr.3.

56 WLB Cod.jur. $2^{\circ}$ 308,3-24. Mai 1603. Vgl. Adam (wie Anm. 4, Bd.2) S. 453 f., Anm. 2.

57 Hertel (wie Anm. 8) S. 120.

58 HStAS A 58 Bü 28, Nr. 2 (Contract des Seidinhandels halb, $\mathbb{S} 12$ ); HStAS A 58 Bü 28, Nr. 4, 10 ff., 14, 18; HStAS A 58 Bü 28, Nr. 26; HStAS A 58 Bü 28, Nr. 10.

59 Weckherlin datierte sie fälschlicherweise in das Jahr 1603, womit sie das erste Geschäftsjahr abdeckte; Ders. (wie Anm.3) S.129.
} 
werden. Hiervon gebührte dem Herzog vertraglich der neunte Teil, also $105 \mathrm{fl}$. $24 \frac{1}{2} \mathrm{kr}$. Für die sieben Mitinteressenten blieben $843 \mathrm{fl} .15$ 1/2 kr. Die Rechnung teilt nun durch acht, womit sich je 105 fl. 20 kr. ergeben. Da Cäsar Morell nur mit $1.000 \mathrm{fl}$., statt mit $2.000 \mathrm{fl}$. beteiligt war, gebührte ihm nur ein halber Anteil (52 fl. 40 kr.). Die zweite Hälfte verteilte sich auf die übrigen Mitinteressenten, sodas diese einen Gewinn von 112 fl. 51 kr. erzielten. Wenn man nun alle Posten zusammenrechnet, die dem Herzog zustanden, hätten an Friedrich I. eigentlich $363 \mathrm{fl}$. $71 / 2 \mathrm{kr}$. aus dem Bargeldbestand ausgezahlt werden müssen. Tatsächlich zahlte Herzog Friedrich I. aber aus seiner eigenen Kasse drauf. Die Rechnung im seydinhandel sine dato führt nämlich als nächsten Posten Gannsen und Cäsars besoldung $380 \mathrm{fl}$. - Item lautt bey gelegten irer Fürstlichen Gnaden bevelch zu gevattergellt $18 \mathrm{fl}$. auf. In Summe macht dies $398 \mathrm{fl}$., abzüglich des fürstlichen Guthabens von $363 \mathrm{fl} .7 \frac{1}{2} \mathrm{kr}$. ergibt sich ein negativer Endbetrag von $34 \mathrm{fl} .52 \frac{1}{2} \mathrm{kr}$. so durch die lanndtschreiber zuerstatten ${ }^{60}$.

Zusammenfassend ergibt die Rechnung im seydinhandel sine dato folgendes Bild: Während die Mitinteressenten in dem fraglichen Jahr immerhin eine gewisse Rendite von etwas über $5 \%$ ihres eingesetzten Kapitals ausbezahlt bekamen, musste Herzog Friedrich durch außervertragliche Faktoren noch draufzahlen: zum einen weil die Besoldung der beiden Geschäftsführer des Stocks Wolfgang Gans (190 fl.) und Cäsar Morell (190 fl.) nicht aus dem allgemeinen Handelsaufkommen, sondern aus dem herzoglichen Anteil erfolgte, was bis 1606 übliche Praxis war, und zum zweiten weil Herzog Friedrich verschiedenen Mitarbeitern im Stock ein sogenanntes gevattergeltt bewilligte. Damit stellte das vierte Geschäftsjahr einen Wendepunkt in der Entwicklung der Handelskompanie dar, denn in den vorhergehenden Jahren konnte Herzog Friedrich wenigstens einen kleinen Gewinn (ad manum) erzielen: 182 fl. 59 kr. im zweiten Geschäftsjahr (23. April 1603 bis 23. April 1604) ${ }^{61}$ und $230 \mathrm{fl} .17 \mathrm{kr}$. im dritten Geschäftsjahr (23. April 1604 bis 23. April 1605 ${ }^{62}$. Für den Gewinneinbruch im vierten Geschäftsjahr macht eine Underthenige relation chammermeisters die abgehörtte rechnung des seidenhandels im stockh von georgii 1605 bis 1606 bethreffendt zwei konkrete Ursachen aus: das Euer Fürstliche Gnaden die drey erste jahr, die maister und maisterin sampt den kündern in dero costen ainig erbalten, und dannenbero die ausbeutten desto höher und sterckher gewesen. Dargegen würdt in dieser rechnung über die 700 fl. eingebracht, so allain über die besoldungen den laboranten, und costgelter der künder gangen. So solle auch bey mansgedenckben die robe seiden nie theurer als an yetzo gewesen sein, dan man heurigs jabrs zu Venedig, das pfund solicher seiden gleich ankauffs umb ein cronen theurer als beim anfang dises handels annebmen müessen,

60 HStAS A 58 Bü 28, Nr. 7.
61 HStAS A 58 Bü 28, Nr. 4.
62 HStAS A 58 Bü 28, Nr.7. 
weliches allain an den zwoen in disem jabr ausgearbeitten ballen, bis zu die $600 \mathrm{fl}$. belaufft $t^{63}$.

Als unternehmensinterne Ursache nennt die relation chammermeisters die $\mathrm{ab}$ dem vierten Geschäftsjahr durch die Kompanie zu erfolgenden Besoldungen von Meister und Meisterin im filatorio (die in den Quellen nicht weiter greifbar sind) sowie die Kostgelder der Armen Kinder. Die Städte und Ämter, die sich ja mit $20 \mathrm{fl}$. pro Kind an den Unterhaltskosten der Kinder beteiligen sollten, waren mit diesem Arrangement von Anfang an nicht einverstanden, was sie in einer gemeinsamen Erklärung kundtaten ${ }^{64}$. Davon unbeeindruckt erwiderte Herzog Friedrich in einem Befehlsschreiben: Weil wir dann derselbigen nunmebr notturffig, so ist hiemit unser bevelch, ir wöllen solche bede kinder fürderlich biebero zu unsern verordneten bemellts Seydinwerk abfertigen ${ }^{65}$. Die Primärintention Herzog Friedrichs geht hier klar hervor - der Herzog brauchte die Kinder als billige Arbeitskräfte für sein Seidenwerk, deshalb sollen sie kommen. Genau dies dürfte gleichzeitig der große Kritikpunkt der Städte gewesen sein, dass sie nämlich Herzog Friedrichs gewinnorientiertes Seidenunternehmen nicht subventionieren wollten, wobei die Aufrichtigkeit der zu Grunde liegenden Prämisse, den Kindern zu einem Broterwerb verhelfen zu wollen und Kenntnisse in der Seidenweberei unter das Landvolk zu bringen, keineswegs ausgeschlossen war. Aber wie stichhaltig war dieses Argument wirklich?

Im Verlauf des vierten Geschäftsjahres, dem Jahr also, in dem die Versorgungspflicht der Armen Kinder vertragsgemäß vom Herzog auf die Handelskompanie überging, liefen von den 53 Kindern insgesamt 37 davon ${ }^{66}$. Zwei der Kinder rechtfertigten sich unter kläglichem wainen, dass sie in Stuttgart nichts lernen würden, noch nicht einmal eine Suppe zu kochen. Flehentlich baten sie darum, nicht nach Stuttgart zurückgeschickt zu werden ${ }^{67}$. Sogar in einer offiziellen Verlautbarung hieß es, als die Kinder fortgelaufen waren: So haben sie jedoch nicht allein wenig

${ }^{63}$ HStAS A 58 Bü 28, Nr. 27.

${ }^{64}$ Die Erklärung, auf die in HStAS A 58 Bü 28, Nr. 2 Bezug genommen wird, ist nicht Teil der Überlieferung, was auch im Directorium dazu explizit erwähnt wird. Da aber Herzog Friedrich seine Anordnung aus dem Generalreskript vom 13. November $1601 \mathrm{mit}$ dem Befehlsschreiben (A 58 Bü 28, Nr.2) nachdrücklich betonen musste, kann man schwerlich von einer positiven Rezeption des Generalreskripts ausgehen. Dennoch schrieb Weckherlin: „Da es unverkennbar war, daß durch eine solche Einrichtung neben dem Nutzen der Fabrik auch für das gemeine Wesen nicht unbedeutende Vortheile erreicht, die Armenkästen erleichtert - die Kinder vom Müssiggang abgehalten und praktisch in einer Kunst unterrichtet werden sollten, wodurch die Verbreitung des Seidenbaues unter die kleineren Landwirthe künftig sehr erleichtert würde, so nahmen viele Städte und Aemter das Anerbieten mit Dank auf ..." (wie Anm. 6, S. 123). Diese Einschätzung spiegelt wohl eher fürstliches Wunschdenken wider. Vgl. Adam (wie Anm. 4, Bd.2) S. 453.

${ }^{65}$ HStAS A 58 Bü 28, Nr. 2 .

${ }^{66}$ HStAS A 58 Bü 28, Nr. 24, 26, 29.

${ }^{67}$ HStAS A 58 Bü 28, Nr. 13 a. 
gelernt, sonder seyen auch mebrertails über des leichtfertiger waiß uffgedretten ${ }^{68}$. Von einer regelrechten Ausbildung der Kinder kann damit keine Rede sein. Nachvollziehbarerweise bekamen die Armen Kinder als schwächstes Glied in der Kette den Kostendruck im vierten Geschäftsjahr als Erstes zu spüren. Als der Herzog und die Städte und Ämter für die Versorgung der Kinder zuständig waren, kamen für die Kinder $1.855 \mathrm{fl}$. im Jahr zusammen, und selbst dieses Geld sollte nur zur underbaltung allerdings, obn all moer ferner zuthun reichen ${ }^{69}$. Die Handelskompanie rechnete in einer Kostenaufstellung gerade einmal mit $360 \mathrm{fl}$. Kostgeld ${ }^{70}$. Die Prämie von einem Batzen für jedes aufgespulte Pfund Seide, mit der die Handelskompanie das Weglaufen der Kinder zu verhindern suchte, musste wie Hohn wirken $^{71}$. Sie fühlten sich zu Recht ausgenutzt. Ganz so müßig scheint ihr weiteres Leben im Übrigen gar nicht verlaufen zu sein, denn in einer Akte heißt es klar, dass die Kinder sich in andere dienstungen oder handthierungen begeben [hätten] ${ }^{72}$. Auch von Seiten der Handelskompanie entscheid man nach dem vierten Geschäftsjahr, auf das System der Armen Kinder weitestgehend zu verzichten ${ }^{73}$.

Als unternehmensexterne Ursache nennt die Relation den um eine Krone pro Pfund gestiegenen Rohseidenpreis in Venedig. Diese Preisentwicklung lässt sich durch eine Kombination aus einem verringerten Angebot an Rohseide bei einer gleichzeitig starken Nachfrage nach Rohseide erklären. Im Zuge des aufkommenden Merkantilismus strebten letztlich alle europäischen Nationen danach, ihren stetig wachsenden Bedarf an Seide durch eine inländische Seidenindustrie abzudecken. Dabei stießen alle Länder nördlich der Alpen im Kern auf die gleiche Problematik unter der auch Herzog Friedrichs Seidenunternehmen litt: Die eigene Rohseidenerzeugung hinkte den Verarbeitungskapazitäten hinterher. Frankreich, England und die Niederlande fanden über ihren Zugang zu den Weltmeeren eine ökonomisch tragbare Möglichkeit, ihren Mangel an eigener Rohseide auszugleichen.

Dabei muss zunächst näher auf die Rolle Venedigs eingegangen werden. Venedig war nämlich in erster Linie als ein Drehkreuz des internationalen Seidenhandels zu verstehen. Zwar verfügte Venedig über eigene Maulbeerplantagen und noch stärker über eigene Seidenwebereien, die breite Masse an Rohseide kam allerdings mit dem Schiff aus den Ländern rund um das Mittelmeer nach Oberitalien, um von dort, verarbeitet oder unverarbeitet, über die Alpenpässe nach Nordeuropa gekarrt $\mathrm{zu}$ werden. Frankreich, England und die Niederlande nutzten die Abwesenheit

68 HStAS A 58 Bü 28, Nr. 14.

69 WLB Cod.jur. $2^{\circ} 308,3-13$. November 1601.

${ }^{70}$ HStAS A 58 Bü 28, Nr.20. Selbst wenn sich diese 360 fl. nur auf die beim Stock verbliebenen 16 Kinder bezieht, gönnte man Ihnen nicht einmal die sprichwörtliche Butter auf dem Brot.

71 HStAS A 58 Bü 28, Nr.24, 26, 29.

72 HStAS A 58 Bü 28, Nr. 14.

73 HStAS A 58 Bü 28, Nr.26, 28. 
Venedigs auf den Handelsrouten im Zuge der Eroberung Zyperns durch die Osmanen 1570/1571 aus, um sich des Zwischenhändlers Venedig zu entledigen und eigene Handelsverträge zu schließen. Handelskompanien dieser drei Nationen, wie etwa die englische Levant Company (gegründet 1592), befuhren nun direkt das östliche Mittelmeer und brachten Rohseide ohne Umwege zu den heimischen Häfen. Vor allem zwischen den Jahren 1604/1605 und 1612/1613 brach der Anteil Venedigs am Handel mit den Anrainern des östlichen Mittelmeers dramatisch ein. Entsprechend verknappte sich das Rohseidenangebot in Venedig. Gleichzeitig versuchten immer mehr Seidenmanufakturen nördlich der Alpen ihren Rohseidenbedarf über den Handelsplatz Venedig abzudecken, und der Preis stieg um eine Krone pro Pfund, was die Gewinnspanne von $178 \mathrm{fl}$. pro Zentner aus der Relation von Wolfgang Gans und Cäsar Morell vom 16. Januar 1602 regelrecht auffraß ${ }^{74}$.

Bereits nach vier Jahren erwies sich das in der Relation erarbeitete theoretische Konzept als praxisuntauglich. Die Seiden-Handelskompanie konnte angesichts eines gestiegenen Rohseidenpreises und des Wegfalls ihres subventionierten Mitarbeiterstabs ihre Gewinnerwartungen nicht mehr erfüllen. Aktenstücke aus dem Jahr 1606 hinterlassen den Eindruck einer geradezu hektischen Suche nach Alternativen $^{75}$. Im Kern kristallisierte sich die Fragestellung heraus, ob man es profitträchtig anstellen könne, statt Rohseide einfach in erster Linie fertige Seidenwaren aus Italien zu importieren und ob sich Cäsar Morell zue solcher sachen erkundigung und einkauffung gebrauchen lassen wölle ${ }^{76}$. Dieser antwortete: Er glaube man khönde alle solche puncten, mit unsers gnedigen fürsten und herrn reputation und deß seidenhandels nutzen, gar wol ins werckh richten. Und da es Ire Fürstlichen Gnaden ime gnedig zuemuethen, wolle er sich darzue inn underthenigkait gern gebrauchen laßen, und darunder seinen müglichsten und eüsersten fleiß anwenden ${ }^{77}$.

$\mathrm{Ob}$ die handels leuthe und krämer ${ }^{78}$ im fünften Geschäftsjahr (23. April 1606 bis 23. April 1607) überhaupt noch Ware aus dem Stock geliefert bekamen, bleibt in den Quellen unbeantwortet und erscheint eher fragwürdig. In jedem Fall ging die Seiden-Handelskompanie in ihrem sechsten Geschäftsjahr (23. April 1607 bis 23. April 1608) selbst zum Einzelhandel über, denn Herzog Friedrich genehmigte ein Anbringen vom 22. Juli 1607 zur Einrichtung eines Kramladens im Stuttgarter

74 HStAS A 58 Bü 28, Nr. 1; Philip Longworth, The Rise and Fall of Venice, London 1974, S. 235-253; Richard Tilden Rapp, Industry and Economic Decline in Seventeenth-Century Venice, Cambridge/London 1976, S.18-20, 96, 105; Luca Molà, The Industry of Renaissance Venice, Baltimore 2000, S. 55-88; Pfister, Art. Seide (wie Anm. 2) Sp. $1049 f$.

75 HStAS A 58 Bü 28, Nr. 20, 22, 23, 24, 25, 26, 29.

${ }^{76}$ HStAS A 58 Bü 28, Nr. 22.

77 HStAS A 58 Bü 28, Nr.23.

78 WLB Cod.jur.2 $308,3-24$. Mai 1603. 
Stockgebäude für 312 fl. ${ }^{79}$ Die Handelskompanie wandelte sich also von einer Seidenmanufaktur mit angeschlossenem Großhandel hin zu einem Einzelhändler für Importseidenwaren mit sporadischer Eigenproduktion (Landseide). Tragischerweise erlebte es Herzog Friedrich nicht mehr, dass die Landschreiberei erstmals einen direkten Gewinn aus der Handelskompanie verzeichnete. Erst unter seinem Sohn und Nachfolger Herzog Johann Friedrich findet sich in der Landschreibereirechnung 1608/1609 der Eintrag: Item ix C lviii [958] fl. $12 \mathrm{kr}$. von den verordneten im stockh empfangen, so unserm gnädigen fürsten und herrn uf Georgii 1609. Als damalen die seidinhandels rechnung abgebört und ausgebeütet, zum 9 theil, ausbeüt, und anderm zugetheilt worden, thuot laut zedels ix Clviii fl. $12 \mathrm{kr} .{ }^{80}$ Dem Anschein nach schickte sich das neue Konzept an, durchaus etwas Geld in die chronisch klamme fürstliche Kasse zu spülen. Unmittelbar sah Herzog Johann Friedrich jedenfalls keine Notwendigkeit, an der Richtung, die die Handelskompanie noch unter seinem Vater eingeschlagen hatte, etwas zu ändern ${ }^{81}$.

Dennoch bahnten sich erneut fundamentale Umwälzungen im ursprünglichen Konzept einer auf acht Mitglieder angelegten Kompanie an. Ein Gültbrief vom 3. Juni $1610^{82}$ nennt bereits eine geänderte Struktur der Interessenten des Seidenbandels im Stock. Wolfgang Gans war nicht mehr Teil der Kompanie, dafür traten drei neue Männer aus dem Umfeld des württembergischen Hofs ein. Wenn die drei neuen Mitinteressenten gute Renditechancen witterten, wurden sie nicht enttäuscht. In den Landschreibereirechnungen spiegelt sich um 1610 ein gewisses Aufblühen des Stocks durchaus wieder ${ }^{83}$. Durch die Einlagen der neuen Interessenten,

79 HStAS A 58 Bü 28, Nr.30. Vgl. Hertel (wie Anm. 8) S.120; Sauer, Herzog (wie Anm.1) S.214f.

${ }^{80}$ HStAS A 256 Nr. 95, fol. 149 r. Vgl. Adam (wie Anm. 4, Bd. 3) S. 537, Anm. 3.

81 HStAS A 58 Bü 28, Nr. 19. Der neue Herzog wünschte lediglich, das doctor Beer inn solchem handel nicht nur alls ein bloßer factor, sonder mitadministrator deßselben, (inmaßen es der contract ohne dz mit sich bringt) nicht weniger alls die andere bede, gebraucht [...].

82 Adam gibt den Inhalt dieses Gültbriefs in einer Fußnote an; Ders. (wie Anm.4, Bd.3) S. 442, Anm.3. Als Quelle erwähnt er lediglich, dass sich das Original im Ständischen Archiv befinde.

83 Adam (wie Anm.4, Bd.3) S.537, Anm.3 fielen „auf das Neuntel des Herzogs auf Georgii 1609958 fl., [und auf] Georgii 16101.600 fl.“. Während die Einnahmen vom Sankt Georgstag 1609 bei einer eigenen Sichtung der Landschreibereirechnungen in der Rubrik Empfanng inn gemeinn ausfindig gemacht werden konnte, ließ sich beim Sankt Georgstag 1610 der entsprechende Eintrag nicht auffinden. Womöglich wurden die $1.600 \mathrm{fl}$. Gewinn an anderer Stelle in der Landschreibereirechnung verzeichnet und nicht bei dieser Rubrik? Die Zahl von $1.600 \mathrm{fl}$. wirkt dennoch plausibel. Bei der eigenen Sichtung konnte nämlich für den Georgstag 1611 in der Rubrik "Empfanng inn gemeinn" der Landschreibereirechnung 1610/11 der folgende Eintrag ausgemacht werden: Item ii M i C li [2.151] fl. $24 \mathrm{kr}$. von den verordneten im stockh empfanngen, so uff Georgy 1611. Unserm gnädigen fürsten und herrn zu dero ußbeuth, unnd anderer geburnnis. Der orthen gefallen, laut ubrkbunndts ii $M$ i C li fl. 24 kr. (HStAS A 256 Nr. 97, fol.146r). Der herzogliche Gewinn aus der Seiden-Handelskompanie hätte sich demnach zwischen 1609 und 1611 von Jahr zu Jahr um rund $500 \mathrm{fl}$. erhöht, was für die übrigen Mitinteressenten ähnliche Schlüsse zulässt. 
Einlagenerhöhungen der alten Interessenten und allgemeinem Verzicht auf Gewinnausschüttung ${ }^{84}$ erhöhte sich die Kapitalbasis der Handelskompanie bis 1611 auf stolze $52.200 \mathrm{fl} . .^{85}$

Abrupt geschah nun etwas, was zumindest im Oktober 1610 noch nicht auf der Agenda stand ${ }^{86}$; Herzog Johann Friedrich löste bis zum 23. April 1611 alle Mitinteressenten aus ${ }^{87}$. Genaueres ist nur in Bezug auf Benjamin Bouwinghausen von Wallmerode bekannt. Bouwinghausen trat dem Herzog seinen Anteil von $3.000 \mathrm{fl}$. zur Begleichung seiner Schuld an Mattheus Enzlin ab, die durch dessen Urfehde auf den Herzog übergegangen war ${ }^{88}$. Die nächsten zweieinhalb Jahre führte Herzog Johann Friedrich das Seidenhandelsgeschäft als alleiniger Interessent weiter. Über diesen Zeitraum gibt ein abschließender Bericht wie es sich mit dem seidenhandel im stock verhallte, des Kammersekretärs Jakob Rathgeb vom 5. Dezember 1614 Auskunft $^{89}$. Dem Bericht zufolge erzielte der Seidenhandel aus den $52.200 \mathrm{fl}$. Kapital ${ }^{90}$ in den zweieinhalb Jahren vom 23. April 1611 bis zum 11. November 1613 den stattlichen Ertrag von 24.790 fl. Abzüglich 9.294 fl. an Kosten lief dies auf einen beträchtlichen Gewinn von 15.496 fl. und eine Rendite von fast 30 \% in dem fraglichen Zeitraum (2 1 12 Jahre) hinaus. Monetär war Herzog Johann Friedrich mit diesem Überschuss zwar endlich in der Gewinnzone, etwas stimmte aber offenbar nicht, denn der Seidenhandel lief nominell ja nur noch bis zum 11. November 1613, endete also ein halbes Jahr vor dem im Contract des seydinhandels halb offiziell vorgesehen Schlusspunkt. Jakob Rathgeb bringt den Grund zu dieser Entscheidung mit der Formulierung: wo schon die handthierung im stockh, weil sie iedermann inn die augen sticht, allerdings abgeschafft gehört, auf den Punkt. Die Stuttgarter handelsleuthe[n] unnd krämer[n] standen dem staatlich privilegierten Handelshaus, das sich bis zur Unkenntlichkeit von seiner ursprünglichen Konzeption einer Handelskompanie entfernt hatte, zu feindselig gegenüber. Der beste Kunde des Seidenhandels war außerdem immer der württembergische Hof selbst, während der Anklang bei der Stuttgarter Bevölkerung eher gering blieb ${ }^{91}$. Zwar konnte der Hofstaat so eine gewisse Unabhängigkeit gegenüber dem konkurrierenden

${ }^{84}$ HStAS A 58 Bü 28, Nr. 25, 29.

85 HStAS L 5 Bd. 45 fol. 419v. Vgl. Adam (wie Anm. 4, Bd. 3) S. 442.

${ }^{86}$ Das Decretum - seydinhandel im stockb betreffendt - wie die verbesserungsmittel ins werck zu richten erwähnt ein solches Vorgehen nicht (HStAS A 58 Bü 28, Nr. 19).

${ }^{87}$ HStAS L 5 Bd. 45 fol. 419 r f.; Adam (wie Anm.4, Bd.3) S. 442. Vgl. Adam (wie Anm. 4, Bd.2) S. 453 f., Anm.2; Weckherlin (wie Anm.3) S.129; Hertel (wie Anm.8) S.120; Sauer, Herzog (wie Anm.1) S.214f.

88 HStAS A 256, Nr. 97, fol.1137v; Adam (wie Anm. 4, Bd.3) S. 442, Anm. 3.

${ }^{89}$ HStAS L 5 Bd. 45, fol. 419 r-425 v; Verkürzt bei AdAm (wie Anm. 4, Bd.3) S. 442-445.

90 An einer Stelle in seiner Edition der Württembergischen Landtagsakten fasste Adam die Summe fälschlicherweise als eine Schuld gegenüber den Gebrüdern Pireth, italienischstämmigen Händlern mit Sitz in Stuttgart, auf; Ders. (wie Anm. 4, Bd.3) S. 434, Anm. 2.

${ }_{91}$ Man verstand es nicht, sich durch Geschenke und Leihgaben allerhandt leuth wol affectioniert [zu] machen. 
Handelshaus der italienischstämmigen und katholischen Gebrüder Pireth waren, dennoch fiel die Entscheidung, dass der gewin denn caminfeegern oder andern $u ß l e n d i s c h e n$ unßerer christlichen religion widrigen und feindt viellieber gegundt werden sollte ${ }^{92}$.

Jakob Rathgeb bestätigt auch klar den Eindruck, den die Rechnung im seydinhandel hinterlässt, dass bis 1611 in erster Linie die Mitinteressenten aus der Handelskompanie ihren finanziellen Vorteil ziehen konnten. Zwar besaß der Schirmherr Herzog Friedrich I. zweifelsohne ein noch größeres Interesse am Gelingen der Seiden-Handelskompanie als die übrigen Interessenten, es ist dennoch schlicht nicht nachvollziehbar, warum der Herzog sämtliche finanziellen Mali auf sich nehmen sollte. Am deutlichsten wird dieser Punkt an den Besoldungen der beiden Geschäftsführer Wolfgang Gans und Cäsar Morell, die der Herzog als außervertragliche Verpflichtung aus seinem Anteil heraus bezahlte.

Zusätzlich bediente man sich ab 1606 noch eines billigen Taschenspielertricks, um zukünftig den herzoglichen Gewinn aus der Handelskompanie größer erscheinen zu lassen, als er es in Wirklichkeit war. Die Besoldung erfolgte nämlich fortan nicht mehr aus dem herzoglichen Anteil, sondern direkt aus der Landschreibereikasse, wie es bei Dr. Eberhard Beer schon die ganze Zeit gehandhabt wurde ${ }^{93}$. Unter Einschluss dieser Verbindlichkeiten reduziert sich etwa der Gewinn von 958 fl. vom 23. April 1609 um rund zwei Drittel ${ }^{94}$. Aber waren die Gehälter beim Stock überhaupt gegenüber der erbrachten Leistung angemessen? Am 20. Januar 1618 führte der Landschaftliche Kleine Ausschuß auf einer Liste mit Einsparmöglichkeiten an die fürstlichen Komissare den folgenden, zwölften Punkt auf: Obwobl der handel im Stock, usgenommen des seidenspinnens, abgeschafft sein soll, so vernemmen wir jedoch, dass die starke besoldungen der daselbst gebrauchten diener nichts destoweniger iren fürgang, und zwar auch bei denenjenigen behalten, die sich bei disem handel nit übel befunden, sondern nachdem sie mit schlechter

92 Mit caminfeegern bezeichnete die Württembergische Landesordnung von 1552 wälsche Krämer. Vgl. Reyscher (wie Anm. 38) S. 212; Adam (wie Anm.4, Bd.3) S.434, Anm. 2.

93 Diese Änderung wurde in einem Underthönig anbringen verbesserlicher anstellung des seydinhandels als im Stockh betreffend (HStAS A 58 Bü 28, Nr.26) angedacht. Herzog Friedrichs Randkommentar ist sehr schwer zu lesen; er scheint diesem Punkt aber zugestimmt zu haben. Der Bericht wie e $\beta$ sich mit dem seiden handell im Stockh verhallte des Kammersekretärs Rathgeb vom 5. Dezember 1614 erwähnt zudem, dass jeder der drei Männer aus der Landschreiberei entlohnt wurde. Insofern erfolgte die Änderung letztlich in jedem Fall. Vgl. HStAS L 5 Bd. 45, fol.419v; AdAm (wie Anm.4, Bd.3) S. 442. Cäsar Morell nutzte die Situation im Jahr 1606 noch zu seinem eigenen Vorteil aus, indem er für sich das stattliche Gehalt von $320 \mathrm{fl}$. als Gegenleistung für seine Dienste beim Einkauf von Seidenwaren in Italien erwirkte. Vgl. HStAS A 58 Bü 28, Nr.23, 26; АDAm (wie Anm.4, Bd.3) S. 442 f., Anm. 5 .

${ }^{94}$ HStAS A 256 Nr.95, fol.149r. Auch Adam sieht die herzoglichen „Gewinne“ als „Selbsttäuschung, weil wesentliche Ausgaben nicht vom Seidenhandel, sondern von der Landschreiberei bezahlt wurden." Ders. (wie Anm. 4, Bd.3) S. 537, Anm.3. Neben weiteren Personalausgaben ließen sich auch sächliche Ausgaben anführen. 
substanz bier ankommen, sich anjetzo umb ein nambaftes gebessert und (darzu noch obne einige verrichtung) stattliche leibgeding baben ${ }^{95}$. Es braucht nicht viel Phantasie, um in der zitierten Quelle eine Spitze gerade auf Cäsar Morell und Wolfgang Gans zu lesen, die beide nicht aus dem Herzogtum Württemberg stammten und als Mitinteressenten der Handelskompanie gute Renditen erzielten. Die Gründung einer Handelskompanie erwies sich damit aus monetärer Perspektive für Herzog Friedrich I. von Württemberg als Reinfall und er wäre besser bedient gewesen, das Kapital in Gänze selbst zu erbringen, wie er es bei der Seidenraupenzucht ja auch tun musste.

\section{Die Seidenraupenzucht}

Offenbar gingen am 14. April 1602 die ersten 7 lib. 11 lot selbst erzeugter Rohseide zur Weiterverarbeitung beim Stock ein ${ }^{96}$. Wie bei der Seiden-Handelskompanie zeigt sich auch bei der Seidenraupenzucht, dass sich die Verfertigung im Stock erst einmal konsolidieren musste. Als man einen Teil der Landseide nämlich schwarz färben wollte, ging dies so sehr schief, dass dem Laboranten $30 \mathrm{fl}$. vom Lohn abgezogen wurden. Zum Glück hatte man die drei Pfund für Benjamin Bouwinghausen von Wallmerode schon vorher beiseitegelegt ${ }^{97}$. Im Contract des seydinhandels halb sprach sich Herzog Friedrich den alleinigen Nutzen aus seiner selbst erzeugten Rohseide zu, was in den folgenden Jahren darauf hinauslief, dass er seine Landseide der Seiden-Handelskompanie zu den laufenden Preisen überließ3 ${ }^{98}$. Während der nächsten drei Jahre (1603-1605) war die Menge an selbst erzeugter Rohseide allerdings minimal, obwohl aus den Akten klar hervorgeht, dass man in jedem Jahr versuchte, Rohseide zu erzeugen. So bezahlte die Handelskompanie dem Herzog 1603 gerade einmal 2 fl. 34 1 12 kr., was auf nicht einmal ein halbes Pfund erzeugter Seide hindeutet ${ }^{99}$. Mit dem Ende des ursprünglichen Geschäftsmodells der Seiden-Handelskompanie und der Suche nach Alternativen dürfte auch die eigene Seidenraupenzucht wieder in den Fokus gerückt sein. So ist zumindest zu erklären, dass 1606 ein Meister über die seiden würm namens Jacomo di Marchit in den Quellen auftaucht ${ }^{100}$. Dieser neue Meister konnte aber auch keine

95 Adam (wie Anm.4, Bd.3) S. 537. Mit dem Begriff „diener“ bezeichnete schon Jakob Rathgeb in seinem Bericht vom 5. Dezember 1614 das zum Stock gehörige Personal in seiner Gesamtheit (allß Doctor Bebren, Ganßen, Cäsar Morell, denn ladendienern, Italienern unnd andern).

${ }^{96}$ HStAS A 58 Bü 28, Nr. 8 .

97 HStAS A 58 Bü 28 , Nr. 4 .

98 HStAS A 58 Bü 28, Nr. 17. Vgl. Weckherlin (wie Anm. 3) S. 126.

99 HStAS A 58 Bü 28, Nr. 19.

100 HStAS A 58 Bü 28, Nr.15. Vgl. Adam (wie Anm. 4, Bd. 2) S. 453 f., Anm. 2; Weckherlin (wie Anm.3) S. 122; Hertel (wie Anm. 8) S.120; Sauer, Herzog (wie Anm. 1) S. 214. 
Wunder vollbringen und so lieferte er dem Stock 1606 nur 11 lib. 11 1/2 lot, die einem Gegenwert von 44 fl. 25 kr. entsprachen, sowie 1607 noch einmal 11 lib. 11 1 1/2 lot. Ernüchtert kommentierte Herzog Friedrich die Quittung des Jahres 1606 mit den Worten: is wenig ${ }^{101}$. Auch Herzog Johann Friedrich zeigte sich in einem Dekret vom 10. Oktober 1610 über die eigene Rohseidenproduktion enttäuscht ${ }^{102}$.

Das Problem lag nicht in der Art und Weise der Aufzucht der Seidenraupen, sondern ging an die Substanz. Am 1. Juni 1604 erließ Herzog Friedrich folgendes Generalreskript an den Untervogt zu Urach: Die weil wir zu erhaltung unserer seyden würm allbie maulbeer bäum laub haben müeßen; und dann der maulbeer baum etliche in deiner ambtung enthalten: So ist unser befelch, $d u$ wöllest zaigern diß, unsern beiden gärttnern, amtshalben verholfen seyn, daß sie alsobald und obn verzogenlich in deiner anbefoblenen ambtung, so es dergleichen bäum hat, daßelbe laub abbrechen: und allhero bringen mögen; und wöllest $d u$ mit denenjenigen, denen solche bäum zu gebören, handlen, daß sie uns solch laub in billichem ohngestaigerten webrt widerfahren laßen; auch wöllest $d u$ denn beiden gärttnern, wo sie über nacht bleiben müeßen, notbwendige zäbrung, doch obne allen überfluß, raichen, und zu allher fübrung solches laubs in säcken, ibnen ein pferdt um gebührende belohnung bestellen, und un $\beta$ beneben berichten, wie viel nicht allein jeder sackvoll maulbeer laub dem getrofenen geding nach; sondern auch das pferdt lohn koste, und was die beide prucht für zährung aufgewendet haben, damit wir dir alsdann darauf der bezablung halber fernern beschaidt ertheilen khenden daran ${ }^{103}$. Es fehlte in Stuttgart also ganz offenbar am Futter für die Seidenraupen, sprich an genügend Maulbeerlaub ${ }^{104}$. Die Seidenraupe muss während ihres rund 35 Tage dauernden Larvenstadiums zwingend alle für ihre Metamorphose benötigten Nährstoffe aufnehmen. Das monophage Insekt frisst deshalb täglich sein Körpergewicht an Maulbeerblättern. Gerade die konstante Nahrungsversorgung ist für eine erfolgreiche Seidenraupenzucht unerlässlich, sonst leiden in jedem Fall Qualität und Quantität des Gespinstes ${ }^{105}$.

101 HStAS A 58 Bü 28, Nr.15, 16, 17.

102 HStAS A 58 Bü 28, Nr. 19: Was nun erstlich Irer Fürstlichen Gnaden aigne landtseidin betrifft, befinden Ire Fürstliche Gnaden das derselbigen wenig gnug, dannenhero Iren Fürstlichen Gnaden ein usfürlich bedencken, wie solche zuvermebren, und zu beßerm eintrag zurichten, durch gedachten renntcammerrhat Moser fürderlich gegeben werden soll.

103 WLB Cod.jur. $2^{\circ}$ 308,4 - 1. Juni 1604.

104 Vgl. Adam (wie Anm.4, Bd.2) S. 453 f., Anm.2; Weckherlin (wie Anm.3) S.129; Hertel (wie Anm. 8) S.120; Sauer, Herzog (wie Anm. 1) S. 214 f.

${ }^{105}$ H. Nicolai, Leitfaden für den deutschen Seidenbauer. Aus der Praxis für die Praxis. Eine Anweisung, den Seidenbau nutzbringend zu betreiben, Berlin [um 1928], S.72; C.H. Pathe, Das Ganze der Maulbeerbaumzucht. Nebst Anleitung zum Seidenbau vom Samenkorn bis zum Seidenfaden, Berlin 1860, S.63 ff.; Werner von RHEIN, Deutscher Seidenbau (Arbeiten der Deutschen Gesellschaft für Züchtungskunde, Bd.67), Hannover 1935, S. 32-39; Rudolf Mell, Der Seidenspinner (Die Neue Brehm Bücherei. Das Leben der Tiere und Pflanzen in Einzeldarstellungen, Bd.34), Leipzig 1951, S. 30. 
Die Problematik, über die Stuttgarter Maulbeerplantagen nicht genügend Maulbeerblätter zur Schlüpfzeit der Seidenraupen zu bekommen, zieht sich durch den gesamten Zeitraum des fürstlichen Seidenunternehmens. Noch in einem Brief vom 5. Mai 1624 an Herzog Johann Friedrich berichtet der neue Geschäftsführer der Seidenraupenzucht Johann Schott: vonn Johann Kormessern maulbeerbohm gärttnern ist mir diser tagen angezaigt worden, wie das der spanische unnd hirlenndische Seiden worm sabmen im ussbeissen sich also erzaigen: unnd er sovil verspüren thue, das wann $d z$ wetter allso, wie es bißhero gewesen, vortsetze, er sovil seidenn würm bekhomm, das er mit albirigem maulbeehr laub selbige nit nach notturfft unnd obne schadenn abzuspeissenn getrawe, unnd weill hievor in gleichem fabl seidenn würm nacher Böblingen im schlo $\beta$ in ein gemach neben einem albie angenommenen mägdtlen selbigen abzuwarthenn verordnet wordenn, also füelltth er inn underthenigkeit darfür das hewerigs jar ein guotte anzabl wurm neben einem mädtlenn nacher Böblingen transferiert, unnd das laub vonn denn maulbeebr bohmenn zu herrenberg albie geführt wirde ${ }^{106}$. Dem Seidenunternehmen kam es hier zugute, dass Herzog Friedrichs Bemühungen um die Pflanzung von Maulbeerbäumen, man denke an Jakob Rathgebens Seydenwurm-Traktat, durchaus fruchteten. Jakob Rathgebens Abschlussbericht zur Seidenhandelskompanie spricht von vil tausend bin und wider und anhand der beiden oben zitierten Quellen lassen sich ältere Maulbeerpflanzungen mit Bestimmtheit nach Urach und Herrenberg verorten, und auch für Böblingen sind Maulbeerbäume ab circa 1606 belegt ${ }^{107}$. Gleichzeitig soll nicht der Eindruck entstehen, es wäre in der Frühen Neuzeit jemals wirklich geglückt, den Seidenbau in Württemberg so heimisch zu machen wie den Weinbau $^{108}$. Aber was war die tiefere Ursache? Warum konnte in Stuttgart allein nicht genügend Maulbeerlaub zur Schlüpfzeit der Seidenraupen geerntet werden?

Aufschluss gibt ein Brief des Geschäftsführers der Seidenraupenzucht Johann Schott vom 23. Januar 1624 an Herzog Johann Friedrich. In diesem Brief schrieb Johann Schott in Bezug auf die jungen Maulbeerbäume: allda es etwas wüntterigs, und thuet durch die früelings gefrössten gar schwerlich ufzupringen sein, unnd durch einen klainen reiffen dabin geben ${ }^{109}$. Herzog Friedrich begann seine Seidenraupenzucht auf dem Höhepunkt der sogenannten „Kleinen Eiszeit“, ein Begriff der die markante globale Abkühlungsphase zwischen dem mittelalterlichen Wärmeoptimum und dem „modernen Treibhausklima“ bezeichnet. Die Jahresmit-

106 HStAS A 58 Bü 28, Nr.3. Vgl. HStAS A 248 Bü 2438, Nr. 5.

107 Rathgeb (wie Anm. 9) fol.3 r: Inmassen der Augenschein an denen Bäumen / die vor langen Jabren gepflanzt worden. WeCKHERLIN (wie Anm.3) S.125: „Das übrigens auch in Böblingen selbst noch nach 100 Jahren alte Maulbeer Bäume angetroffen worden, erhellt aus einem Bericht des Vogts vom Jahr 1706.“ Auch in den Rechnungen der Jahre 1624 bis 1626 wird Maulbeerlaub aus Böblingen erwähnt. Vgl. HStAS A 248 Bü 2438, Rechnungen $1623-1626$.

108 WLB Cod.jur. $2^{\circ}$ 308,3 - 13. November 1601; Rathgeb (wie Anm. 9) fol. 5 r.

109 HStAS A 58 Bü 28, Nr.1. 
teltemperatur sank in der Größenordnung von 1,5 bis $2,0^{\circ} \mathrm{C}$ ab. Besonders starke Abkühlungen verzeichnete man um 1600 und wieder am Ende des 17. Jahrhunderts ${ }^{110}$. In Mitteleuropa äußerte sich die „Kleine Eiszeit“ in sehr kalten, lange andauernden Wintern und niederschlagsreichen, kühlen Sommern, was die natürliche Vegetationsperiode verringerte. Ein spezieller Effekt dieser Abkühlung lag in einer im Durchschnitt stark erhöhten Anzahl an Frosttagen in den Frühlingsmonaten März, April und Mai, was den Blatttrieb der Maulbeerbäume hemmte und gerade junge Pflanzen in ihrer Existenz bedrohte ${ }^{111}$. Herzog Friedrich hielt sich mit seinem Seideninstitut an die Faustregel, dass die Pflanzung von Maulbeerbäumen und damit die Seidenraupenzucht überall dort möglich sei, wo man Weinbau betreiben kann ${ }^{112}$. Während der Weinbau durch die klimatischen Verhältnisse ebenfalls in Mitleidenschaft gezogen wurde, musste dies auf den Seidenbau noch einmal verstärkt zutreffen, denn es war gerade die kritische Frühlingsphase, die durch die klimatischen Verhältnisse besonders negativ beeinflusst wurde. Bei einem Vergleich mit den Wetterdaten der Jahre nach 1600 lässt sich eine gewisse Korrelation zwischen Frühlingsfrost und Ertrag der Seidenraupenzucht Herzog Friedrichs durchaus feststellen ${ }^{113}$.

Im Gegensatz zur Seiden-Handelskompanie bekam die Seidenraupenzucht eine zweite Chance. Jakob Rathgebens Bericht wie es sich mit dem seidenhandel im stock verhallte gibt zwei Gründe für die Entscheidung an, die fürstliche Seidenraupenzucht weiter zu betreiben. Zum einen wären dann alle aufgewendeten Kosten und Mühen umsonst gewesen und zum anderen hätten die letzten beiden Jahrgänge neue Maßstäbe im Hinblick auf den erwartbaren Ertrag gesetzt. In der Tat konnte man 1613 und 1614 zusammen stolze 74 lib. Landseide erzeugen, die einen Erlös beim Stock von 740 fl. erzielten ${ }^{114}$. Bedeutender als der finanzielle Erlös wiegte allerdings die Aussicht, den Eigenbedarf des Fürstenhofes, der sich in

110 Rüdiger Glaser, Art. Kleine Eiszeit, in: Enzyklopädie der Neuzeit, Bd.6, Stuttgart 2007, Sp. 767-771.

111 Hubert Lamb, Climate, History and the Modern World, London/New York 1982, S. 201-206; Rüdiger Glaser, Klimageschichte Mitteleuropas. 1200 Jahre Wetter, Klima, Katastrophen, Darmstadt 2001, S. 82-87; Christian Pfister, Weeping in the Snow. The Second Period of Little Ice Age-type Impacts, 1570-1630, in: Kulturelle Konsequenzen der „Kleinen Eiszeit“. Cultural Consequences of the „Little Ice Age“, hg. von Wolfgang Behringer/Hartmut Lehmann/Christian Pfister, Göttingen 2005, S.31-86; Reinhold ReIth, Umweltgeschichte der Frühen Neuzeit (Enzyklopädie Deutscher Geschichte, Bd. 89), München 2011, S. 9 ff.

112 Rathgeb (wie Anm.9) fol.3v, 12 v. Kritische Stimmen zur Seidenraupenzucht in nördlichen Ländern gab es durchaus schon zur Zeit Herzog Friedrichs. Vgl. Rathgeb (wie Anm. 9) fol. $3 \mathrm{rf}$.

113 Glaser, Klimageschichte (wie Anm.111) S. 130-137.

114 HStAS L 5 Bd 45, fol. 420 v; Adam (wie Anm. 4, Bd. 3) S. 443. Der Rohseidenpreis war inzwischen auf 10 Gulden pro Pfund gestiegen. Die Relation vom 16. Januar 1602 ging zum Verleich noch von einem Preis für fertige Seidenware von 7 Gulden pro Pfund aus. 
etwa auf 30 bis 40 Pfund pro Jahr beziffern lässt, durch selbst erzeugte Landseide zu decken ${ }^{115}$. Wenn der Seidenhandel während seiner Endphase vom 23. April 1611 bis zum 11. November 1613 noch Landseide von der Raupenzucht annahm, setzt dies natürlich ein Aufrechterhalten der Verarbeitungskapazitäten im Stuttgarter Stockgebäude neben dem Kramladen voraus. Um nur die selbst erzeugte Rohseide für den fürstlichen Eigenbedarf zu verarbeiten, brauchte es jedoch nicht zwingend italienische Facharbeiter, die natürlich einen deutlich höheren Lohn einfordern konnten als einheimische Laboranten ${ }^{116}$. So lässt sich zumindest erklären, dass drei italienische Facharbeiter auf den St. Martinstag (11. November) 1617 entlassen wurden. Dafür tauchen in den späteren Rechungsbüchern deutsche Namen bei der Seidenverarbeitung auf. Auch Jacomo di Marchi’s Funktion als Meister über die Seidenwürmer ging zusätzlich auf den Maulbeerbaumgärtner Johann Kornmesser über - Informationen, die etwas Licht auf den ansonsten quellenlosen Zeitraum nach 1614 werfen $^{117}$.

Für die 1620er Jahre stehen wieder Akten zur Verfügung. Die 1608 angelegte Maulbeerplantage „auf der Baumreute im Eiernest“ war inzwischen wüstgefallen (zue egartten worden), weil die Maulbeerbäume darinnen nicht gerathen wollen, sonder verdorben beziehungsweise darauf nit wachsen wöllen und keinen fortgang haben, sodass der Postbote Hans Hackl das Stück 1623 für 350 fl. kaufen konnte, um es wieder in Ackerland zu verwandeln ${ }^{118}$. Die zwei Stuttgarter Baumschulen das eine hündern Jäger Hauß unnd das ander bey dem Acht Eckbeten Thurn verwendete man inzwischen ebenfalls anderweitig. Als „Mutterland“ für die Maulbeerbäume diente nun ein Grundstück in dem Vichgarttenn, wo der Gärtner Johann Kornmesser im Januar 1624 viereinhalb Tausend junge Maulbeerbäume wartete. Dieses Grundstück erwies sich aber als besonders ungeeignet, die Maulbeerschößlinge angesichts der extremen Wetterverhältnisse aufzubringen. Die geplante Versetzung von eintausend Schößlingen stand durch die Frühlingsfröste auf Messers Schneide. Gewisse Hoffnungen setzte Johann Kornmesser auf ein neues Grundstück im tiergartten [...] bünder dem newen lusthaus ( $1 / 4$ Morgen), das als neue Baumschule dienen sollte ${ }^{119}$. Von diesem Grundstück war man bereits im April 1624 wieder abgerückt. Johann Kornmesser hatte nun ein Grundstück

115 HStAS A 248 Bü 2438: Rechnungen 1623-1626; HStAS A 58 Bü 28, Nr. 10: [...] und füraus alle jar 30 oder 40 pfund seydin, obne bezalung in das fürstlich gewölb zu antworten getrawte $[. .$.$] .$

116 Vgl. eine Liste mit Einsparmöglichkeiten des Landschaftlichen Kleinen Ausschusses vom 20. Januar 1618 (Adam (wie Anm. 4, Bd.3) S. 537), die explizit die starke[n] besoldungen der daselbst [beim Stock] gebrauchten diener ankreidet.

117 Adam (wie Anm.4, Bd.3) S. 537, Anm.3; HStAS A 58 Bü 28; Nr.10; HStAS A 248

Bü 2438: Rechnungen 1623-1626.

118 HStAS A 403 II Bü 21, Nr. $1 \mathrm{ff}$.

119 HStAS A 58 Bü 28, Nr.1. 
an dem Kreuttackher, usserbalb euer fürstlichen gnaden geliebter gemablin gartten (1/8 Morgen) ins Auge gefasst ${ }^{120}$.

Die Flächen für die Aufzucht der Maulbeerbäume wurden zusehends kleiner, womit sich die Seidenraupenzucht in Bezug auf ihre Grundlage, sprich die Verfügbarkeit einer ausreichenden Menge an Maulbeerlaub, auf dem absteigenden Ast präsentiert. Aber für das fehlende Maulbeerlaub aus Stuttgart hatte sich ja eine Ersatzlösung etabliert - und so findet sich das laubrechen als fester, immer wiederkehrender Posten in den drei erhaltenen Rechnungsbüchern aus den Jahren 1624, 1625 und 1626 wieder. Im Mai 1623 brachte allein der vorstmeister von Böblingen Georg Jäger [...] 300 reißach uff dreyen wägen allhero ${ }^{121}$.

So problematisch sich das Ziehen der Maulbeerbäume in Stuttgart auch gestaltete, konnte man anscheinend im Herzogtum genügend Laub auftreiben, sodass sich in den 1620er Jahren die Menge an selbst erzeugter Landseide erstaunlicherweise als relativ stabil auf dem Ertragsniveau erwies, das man in der Endphase der Handelskompanie erreichte und Jakob Rathgeb in seinem Bericht vom 5. Dezember 1614 als erwartbar formulierte: 49 lib. 26 lot im Jahr 1621; 21 lib. im Jahr 1622; 68 lib. 10 lot im Jahr 1623; 77 lib. 20 lot im Jahr 1624; 17 lib. 24 lot im Jahr 1625; 30 lib. 29 lot im Jahr 1626; 23 lib. 2 lot im Jahr $1627^{122}$.

Wirklich zufrieden zeigte sich der Geschäftsführer der Seidenraupenzucht, Johann Schott, mit seinem eigentlich doch mehr als vorzeigbaren Ergebnis indes nicht. Die eingesetzte Menge an spanisch seiden wurm sabmen belief sich 1626 auf 1 lib. 24 lot, was mit dem Ergebnis von 30 lib. Rohseide in keinem Verhältnis stand, da es doch ein nambaffts mebrer unnd uber 100 pfundt geben haben sollte ${ }^{123}$. In einem Brief vom 12. Februar 1627 benannte der Geschäftsführer der Seidenraupenzucht die Hintergründe: das Johann Kornmesser maulbeehr babm garttner, der der maister hierüber ist, mir [Johann Schott] uff besprachen diesen mangel angezaigt, das er khain andere ursach wissen, den das die gemächer im Stockh gar zu verdumpffen sey, unnd wann man die würm hünder dass reiss [Frühstadium der Larve] setze werden sie gantz matt und faul $d z$ sie nit spünnen khönden ${ }^{124}$. Die übermäßige Kälte im Frühling setzte nicht nur den Maulbeerbäumen zu, sondern auch den Seidenraupen selbst. Liegt die Temparatur unter $15{ }^{\circ} \mathrm{C}$ schränkt die Seidenraupe ihre Aktivität in gefährlichem Maße ein, verweigert die Nahrungsaufnahme und häutet sich nicht ${ }^{125}$. Ein Blick auf die Wetterdaten zeigt, dass die Problematik

120 HStAS A 58 Bü 28, Nr. 2.

121 HStAS A 248 Bü 2438: Rechnungen 1623-1626. Vgl. WeckHerlin (wie Anm.3) S. $130 \mathrm{f}$.

${ }^{122}$ HStAS A 248 Bü 2438: Rechnungen 1623-1626; HStAS A 58 Bü 28, Nr. 8, 11.

123 HStAS A 58 Bü 28, Nr. 10.

124 Ebd.

125 Pathe (wie Anm.105) S.54-57; Arnold Voelschow, Die Zucht der Seidenspinner. Ausführliche Beschreibung sämtlicher in Europa eingeführten Seide erzeugenden 
unverändert blieb ${ }^{126}$. Während sich das fehlende Maulbeerlaub aus Alternativquellen beschaffen ließ, konnte dieses Problem nur über die eigene Produktionsstätte angegangen werden. Bereits in einem Brief vom 16. Oktober 1624 an Herzog Johann Friedrich machte Johann Schott das unzureichende Lokal (im Stockh nit weitte und platz gnug), als den begrenzenden Faktor dafür aus, bei der Rohseidenerzeugung einen Zentner zu erreichen. Gleichzeitig präsentierte er einen Lösungsvorschlag als Überschlag zue erbawung eines hauß für die seidenwürm im holtzgarthen gägenüber dem gerber graben ${ }^{127}$. Minutiös werden die einzelnen Posten (maurer arbaitt, zimmer werckh, thönnen holtz, geschnidtner zeng, schreiner arbaitt) zue erbauwung eines hauß für die seidenwürm, so abe der leng halten soll 90 schub unnd an der breite 35 schub mit zweyen stockbwerckhen dz under gantz frey uf etliche steinere pfüller unnd innen aber bültzerne säullen den oberen stockh verrigelt, sambt seinem tachwerckh daruff aufgelistet, die sich in der Gesamtsumme auf 1.333 fl. 28 kr. 3 hlr. beliefen ${ }^{128}$. Johann Schott konnte seinen Lösungsvorschlag durchaus argumentativ unterlegen: da aber Eure Fürstliche Gnaden zway gemächer bauwen unnd in jedes 200 oder 250 taflen sambt zway öfen setzen ließen, allso khöndte die würm nicht allein sommers zeidt vor grosser bitz bewarth, sondern auch wan es ein kalltte früeling gebe, der lufft vermittelt der öfen recht temperiert werden, dardurch er ein nambafft mebrers an seidenn zu wege zupringen getrawte, als das seines underthenig erachtens der bawcost inner 2 oder 3 jabren wider herein gepracht unnd fürtter ein mebrere nutz als bissher beschehen geschafft würde ${ }^{129}$. Dennoch warb Johann Schott mit seinem ersten Brief vom 16. Oktober 1624 und noch einmal am 5. Dezember 1626 und wieder am 12. Februar 1627 dreimal vergeblich um seine Zuchtstation für die Seidenraupen sie wurde nie gebaut.

Der fehlende Wille von Seiten der Obrigkeit unter Herzog Johann Friedrich noch einmal eine dynamische Entwicklung beim Stock zuzulassen, kann aus heutiger Perspektive durchaus als klug und durchdacht bezeichnet werden, denn ihre prinzipielle Aufgabe seit dem Ende der Seiden-Handelskompanie - den fürstlichen Eigenbedarf zu decken - konnte die Seidenraupenzucht auch ohne italienische Facharbeiter, ohne ausgedehnte Maulbeerplantagen in Stuttgart und ohne Zuchtstation für die Seidenraupen erfüllen ${ }^{130}$. So lieferte die Seidenraupenzucht an schwarzer und gefarbet seidin 96 lib. 15 1/2 lot im Jahr 1624 und 72 lib. 151/2 lot im Jahr 1626 in das fürstliche gewölb, wie es aus den drei erhaltenen Rechnungs-

Schmetterlinge und ihre Zucht, Schwerin 1902, S.12f.; Mell (wie Anm. 105) S. 37; NicolaI (wie Anm. 105) S.69.

126 Glaser, Klimageschichte (wie Anm. 111) S.138-145.

127 HStAS A 58 Bü 28, Nr.6. Vgl. Weckherlin (wie Anm. 3) S. 126.

128 HStAS A 58 Bü 28, Nr.7.

129 HStAS A 58 Bü 28, Nr. 10.

130 Einem impulsiv agierenden Herzog Friedrich I. von Württemberg wäre der Bau der Zuchtstation durchaus zuzutrauen gewesen. 
büchern der Jahre 1624, 1625 und 1626 hervorgeht ${ }^{131}$. Es blieben in den Jahren 1621 bis 1626 sogar noch 60 Pfund verfertigter Seide übrig, die wie eine Art Ladenhüter über die Frankfurter Messe verhandlett und verkaufft werdenn muß[ten $]^{132}$. Einen wirklichen Gewinn können besagte 60 Pfund aufgrund der zu hohen Produktionskosten für württembergische Landseide jedoch nicht erbracht haben, wie es Weckherlin bereits vorrechnete ${ }^{133}$. Die in der Zeit vom 23. April 1623 bis zum 23. April 1626 vom Stock an das fürstliche Gewölbe gelieferten Seidenwaren entsprachen einem Gegenwert von 1.754 fl. 54 kr. Gleichzeitig beliefen sich die Gesamtausgaben zur Erzeugung dieser Seidenwaren auf 1.748 fl. 24 kr., so dass von einem finanziellen Vorteil durch die Seidenraupenzucht nicht gesprochen werden kann ${ }^{134}$.

Die Ausgaben setzten sich folgendermaßen zusammen: Außgeben gellt umb seiden würm samen $138 \mathrm{fl} .15 \mathrm{kr}$., Außgeben gellt umb erkauffte species zum ferben $227 \mathrm{fl} .13 \mathrm{kr}$., Außgeben gellt dennjenigen persobnen, so denn würmen abgewartet unnd seiden beraittet 561 fl. 59 kr., Außgeben gellt uff das laubrechen gangen $624 \mathrm{fl} .18 \mathrm{kr}$. und Außgeben gellt inn gemain, die zum größten Teil ebenfalls aus Ausgaben im Zusammenhang mit dem laubrechen bestehen ${ }^{135}$, in Höhe von 196 fl. $38 \mathrm{kr}^{136}$. Die mit Abstand größten Kosten fielen somit im Zuge der externen Beschaffung von Maulbeerlaub an. Damit wird klar, warum Herzog Johann Friedrich zögerte, in den Bau einer zweistöckigen, beheizbaren Zuchtstation zu investieren $^{137}$. Wenn hierdurch mehr Seidenraupen die Frühlingsfröste überlebten war das schön und gut, es gab jedoch über die herzoglichen Maulbeerplantagen in Stuttgart nicht einmal genügend Maulbeerlaub um die bisherigen Seidenraupen abzuspeisen, geschweige denn um die doppelte Menge zu ernähren. Das zusätzliche Maulbeerlaub hätte ebenfalls im Herzogtum aufgetrieben werden und teuer nach Stuttgart gekarrt werden müssen, falls man überhaupt noch zusätzliches Maulbeerlaub hätte auftreiben können. Und wozu das Ganze? Ein Handel mit der zusätzlichen Seide

131 HStAS A 248 Bü 2438. Rechnungen 1623-1626. Vgl. Weckherlin (wie Anm.3) S. $130 \mathrm{f}$. Ferdinand August von Weckherlin gibt die Zahlen nicht vollkommen exakt wieder.

132 HStAS A 58 Bü 28, Nr.10. Nur einmal ist in der Rechnung von 1625/1626 von einem Seidenverkauf außerhalb des fürstlichen schloßgewölbs die Rede, nämlich 3 lib. 11 lot für herrn marggraven zue Durlach geben. dz lb für $8 \frac{1}{2} \mathrm{fl}$. thuet $28 \mathrm{fl}$. $20 \mathrm{kr}$.

133 Weckherlin (wie Anm.3) S.130f. Weckherlin kommt in seiner Addition auf eine leicht unterschiedliche Zahl (1.777 fl. $1 \mathrm{kr}$.) bei den Gesamteinnahmen, in der prinzipiellen Richtung stimmt die hier präsentierte Addition jedoch mit derjenigen Weckherlins überein.

${ }^{134}$ HStAS A 248 Bü 2438: Rechnungen 1623-1626.

135 Zum Beispiel: Alß denn 29. May Anno 1623 der haußschneider zue Böblingen Christoff Jäger daß Maulbeerlaub alhero füehren lassen, hab ich denn fuerleuthen, so drey persohnen gewesenm für ein suppen und trunckh, wie allwegen passiert wordenn, lauth zedels bezalt, leichte wehrung 3 fl. oder Für weisen faden, die seckh, darin mann dz laub bricht zueflickhen erlegt 4 1/2 kr. (HStAS A 248 Bü 2438: Rechnung 1623/1624).

${ }^{136}$ HStAS A 248 Bü 2438: Rechnungen 1623-1626. Vgl. Weckherlin (wie Anm.3) S. $130 \mathrm{f}$.

137 HStAS A 58 Bü 28, Nr.6. Vgl. Weckherlin (wie Anm.3) S.126. 
machte wenig Sinn, weil die fertigen Seidenwaren, durch die hohen Ausgaben für die auswärtige Laubbeschaffung, im Verkauf nur marginal mehr einbringen konnten, als Kosten aufgebracht werden mussten. Ganz davon abgesehen, dass man nach den Erfahrungen mit der Seiden-Handelskompanie von herzoglicher Seite zu Recht vor der Idee eines zweiten Seidenhandels zurückschrecken durfte.

Trotz allem durfte der Stock seine Arbeit bis in die 1630er Jahre hinein fortführen. Den Akten liegt ein zeitgenössischer Abgabevermerk an die Rentkammer über die rechnungen den seydenhandell von Georgii anno 1623 biß Georgii 1632 bei, der als ein erster zeitlicher Orientierungspunkt dienen kann ${ }^{138}$. Als zweiter Orientierungspunkt lässt sich der Zusammenbruch im Zuge der Schlacht von Nördlingen am 6. September 1634 anführen ${ }^{139}$. Für Württemberg fiel die Niederlage der protestantischen Seite in dieser Schlacht fatal aus. Das Land bekam die volle Wucht des Dreißigjährigen Krieges zu spüren, und der Hofstaat musste ins Exil ziehen. An Seidenraupenzucht dachte in diesen turbulenten Zeiten niemand mehr, und so lässt sich das Ende des fürstlichen Seidenunternehmens mit ziemlicher Sicherheit in die Jahre 1632 bis 1634 datieren.

\section{Schlussbetrachtung}

In ihrer Überstürzung ähnelt die Reaktion auf das fehlende Maulbeerlaub bei der Seidenraupenzucht vom Juni 1604 derjenigen der Seiden-Handelskompanie vom September 1606 auf den gestiegenen Rohseidenpreis in Venedig sowie den Wegfall der subventionierten Armen Kinder. In beiden Fällen fand man eine improvisierte Lösung, die perpetuiert wurde. Im Fall der Seiden-Handelskompanie war dies der Verkauf fertiger Seidenware und im Fall der Seidenraupenzucht die Beschaffung des fehlenden Maulbeerlaubs aus allen Ecken des Herzogtums Württemberg. Für eine echte Gewinn- und Verlustrechnung ist die Überlieferungslage zu Herzog Friedrichs Seidenunternehmen zu lückenhaft. Die vorhandenen Geschäftszahlen lassen allerdings nur den Schluss zu, dass weder die Seiden-Handelskompanie (1602 bis 1613) noch die Seidenraupenzucht (1595/1598 bis 1632/1634) die massiven Investitionen aus der Initialphase der Unternehmung jemals wieder einspielten. In jedem Fall erreichte Herzog Friedrich I. von Württemberg mit seinem Seidenunternehmen das oberste Ziel der merkantilistischen Wirtschaftspolitik eine langfristige Sicherung und Mehrung des Reichtums des Staates durch die Erhöhung der Staatseinnahmen - nicht.

Deswegen muss man das Seidenunternehmen jedoch nicht in Gänze aburteilen. In Württemberg erzeugte, verarbeitete oder wenigstens selbst gehandelte Seide besaß einen intrinsischen Wert, den von ausländischen Händlern vertriebene Import-

138 HStAS A 58 Bü 28.

139 Weckherlin (wie Anm.3) S. 129 f.; Adam (wie Anm. 4, Bd.2) S. 453 f., Anm. 2. 
produkte niemals haben konnten. Autarkie hieß die Zielvorstellung der Zeitepoche $^{140}$. Weiterhin blieb noch das Prestige, das Herzog Friedrich mit seiner dynamischen Wirtschaftspolitik auf sich vereinigen konnte. Man denke etwa an die drei Pfund selbst erzeugter Seidenware, die er 1603 seinem Geheimrat Benjamin Bouwinghausen von Wallmerode als Geschenk für den französischen Hof mitgab.

Die Probleme, auf die man in der Praxis stieß, stellen nur die eine, praktische Seite der Medaille dar. Wie gezeigt wurde, repräsentierte das fürstliche Seidenunternehmen die konkrete Umsetzung der merkantilistischen Ökonomie der Zeit Herzog Friedrichs I. Die Hintergründe zum langfristigen Scheitern des fürstlichen Seidenunternehmens müssen daher in enger Verknüpfung mit dem merkantilistischen Theoriegebäude gesehen werden. Der grundlegende Gedankengang war derjenige, den Eli Filip Heckscher als Wandel von einer Versorgungspolitik hin zu einer Schutzpolitik bezeichnete ${ }^{141}$, nämlich den Versuch, sich aus einer Importabhängigkeit durch den Aufbau des entsprechenden Gewerbezweiges, in diesem Fall Seidenbau, zu befreien. Diesem merkantilistischen Gedankengang fehlte jedoch die Überlegung, wie effektiv Seidenbau in Württemberg betrieben werden konnte bzw. zu welchen Kosten. Wie sich nach einigen Jahren herausstellte, bereiteten die klimatischen Verhältnisse erhebliche Probleme bei der Kultur der Maulbeerbäume, und auch die Seidenraupen litten unter den niedrigen Temperaturen. Herzog Friedrichs Seideninstitut befand sich in guter Gesellschaft, denn letztlich scheiterte jeder der zahlreichen Versuche im 17. und 18. Jahrhundert, nördlich der Alpen einen eigenen Seidenbau zu etablieren an den klimatischen Verhältnissen ${ }^{142}$.

Um trotz fehlender Rohseide im Stuttgarter Stockgebäude eine Seidenmanufaktur starten zu können, präsentierten Wolfgang Gans und Cäsar Morell am 16. Januar 1602 eine Lösung, die sich stark an Jean Bodins Außenhandelsthese orientierte, dass ein Import von Rohstoffen und ein Export von Fertigwaren zu empfehlen sei, um die größtmögliche Wertschöpfung im eigenen Land zu erzielen ${ }^{143}$. Konkret sollte die fehlende Rohseide in Venedig eingekauft werden ${ }^{144}$. So raffiniert diese Geschäftsidee auch war ${ }^{145}$, begab man sich damit doch in eine gefährliche Abhän-

140 Rathgeb (wie Anm. 9) fol.2r-5v; Heckscher (wie Anm.13) S.116f.; Gömmel (wie Anm. 13) S. 24; Sokoll (wie Anm. 13) Sp. $382 \mathrm{ff.}$

141 Heckscher (wie Anm. 13) S.116-125. Vgl Gömmel (wie Anm. 13) S. 49 ff. Sokoll (wie Anm.13) Sp. 384 ff.

142 Rhein (wie Anm.108) S.5; Deutsche Seidenbauzentrale (Bearb.), Denkschrift über die bisherige Entwicklung und den heutigen Stand des Seidenbaus in Deutschland nebst Anträgen und Vorschlägen zu seiner weiteren Förderung. Der Reichsregierung überreicht durch die deutsche Seidenbauzentrale e.G.m.b.H. in Sindelfingen, Sindelfingen 1932, S. 1 f.; Molà (wie Anm. 74) S. 63; Pfister, Art. Seide (wie Anm. 2) Sp. 1043 f.

143 Bodin (wie Anm. 48) S. 877.

144 HStAS A 58 Bü 28, Nr.1.

145 Anders als bei der Seidenraupenzucht hätte Adam Smith diesem Geschäftsmodell sofort sein Placet gegeben: „The price of some manufactures would be reduced in a still greater proportion by the free importation of the raw materials. If raw silk could be imported from 
gigkeit des Handelsplatzes Venedig. Zu Beginn des 17. Jahrhunderts standen letztlich alle europäischen Staaten vor dem Problem, nicht genügend Rohseide für ihre Manufakturbetriebe zu produzieren, und alle europäischen Staaten rezipierten Jean Bodins Außenhandelsthese. Württemberg befand sich in dieser Ausgangssituation gegenüber den westeuropäischen Staaten in einem entscheidenden Nachteil, denn während Staaten wie Frankreich, England und die Niederlande über ihren Zugang zur See die fehlende Rohseide direkt an der Quelle - bei den Mittelmeeranrainern - beschaffen konnten, war Württemberg auf den Landweg und somit auf Venedig als Zwischenhändler angewiesen. Wenn die westeuropäischen Staaten die Rohseide der Mittelmeeranrainer aufkauften, konnte sie natürlich nicht mehr in Venedig angeboten werden, und der dortige Preis für Rohseide musste unweigerlich steigen. Genau durch diesen Marktmechanismus verteuerte sich 1605, bereits drei Jahre nach der Gründung der Seiden-Handelskompanie am 23. April 1602, der Rohseidenpreis um eine Krone pro Pfund ${ }^{146}$. Cäsar Morells und Wolfgang Gans’ Geschäftsmodell erwies sich damit als genausowenig nachhaltig, wie Herzog Friedrichs Modell der Armen Kinder. Damit dachte Herzog Friedrich zwei Fliegen mit einer Klappe zu schlagen, nämlich den Kindern eine Weberausbildung anzugedeihen und der Handelskompanie gleichzeitig billige Arbeitskräfte zur Verfügung zu stellen, die auf Subsistenzniveau Hand- und Spanndienste verrichteten. In der Praxis erwiesen sich diese Vorstellungen als unvereinbar, und es lief auf eine reine Ausbeutung der Armen Kinder hinaus. Die Kinder allerdings ließen sich nicht alles gefallen und setzten sich ab.

China and Indostan duty-free, the silk manufacturerers in England could greatly undersell those of both France and Italy. There would be no occasion to prohibit the importation of foreign silks and velvets. The cheapness of their goods would secure to our own workmen, not only the possession of the home, but a very great command of the foreign market." (Adam Smith, An Inquiry into the Nature and Causes of the Wealth of Nations. Two Volumes in One, Chicago 1976, Bd.2, S.416).

${ }^{146}$ HStAS A 58 Bü 28, Nr.27; Longworth (wie Anm.74) S.235-253; Rapp (wie Anm.74) S. 18 ff., 96, 105; Molà (wie Anm.74) S. 55-88; Pfister, Art. Seide (wie Anm. 2) Sp. $1049 \mathrm{f}$. 